\begin{tabular}{|c|l|}
\hline Title & Surface reaction dynamics and energy partitioning \\
\hline Author(s) & Matsushima, Tatsuo; Shobatake, Kosuke \\
\hline Citation & $\begin{array}{l}\text { Journal of Molecular Catalysis. A, Chemical, 315(2), 135-147 } \\
\text { https:/doi.org/L0.1016/.molcata.2009.06.012 }\end{array}$ \\
\hline Issue Date & 2010-01-15 \\
\hline Doc URL & http://hdl.handle.net/2115/42756 \\
\hline Type & article(author version) \\
\hline File Information & J_Mol_Catal_A_2010_Matsushima.pdf \\
\hline
\end{tabular}

Instructions for use 


\title{
Surface reaction dynamics and energy partitioning
}

\author{
Tatsuo Matsushima ${ }^{1}$ and Kosuke Shobatake ${ }^{2}$ \\ ${ }^{1}$ Catalysis Research Center, Hokkaido University, North 21 West 10, Sapporo 001-0021, Japan \\ ${ }^{2}$ Toyota Physical \& Chemical Research Institute, Nagakute, Aichi 480-1192, Japan
}

Corresponding author; Tatsuo Matsushima

Address; Catalysis Research Center, Hokkaido University, North 21 West 10, Sapporo 001-0021, Japan

Tel/Fax; +81-29-874-1508

E-mail; tatmatsu@mbr.nifty.com 
Abstract

New approaches to surface reactions are reviewed in comparison with reaction dynamics in the gas phase. These are commonly based on product analysis before energy dissipation, in contrast to the chemical kinetics of surface reactions. Information of energy partitioning during reaction events is requisite to approach to reaction sites. In reactant adsorption, electronic excitations are exemplified to take place, showing non-adiabatic processes. On the other hand, in product desorption, spatial and energy distributions of desorbing products with hyperthermal energy can deliver the most direct structural information of the transition state including active intermediates and product formation sites. Typical analyses are shown in both $\mathrm{N}_{2} \mathrm{O}$ decomposition and $\mathrm{CO}$ oxidation on noble metals.

Keywords; $\quad$ Surface reaction dynamics, Energy partitioning, Angular distribution, $\mathrm{N}_{2} \mathrm{O}$ decomposition, NO reduction, CO oxidation, Palladium, Rhodium, Platinum

\section{Chemical kinetics and reaction dynamics}

The mechanisms of chemical reactions on solid surfaces have been mostly examined from the viewpoint of chemical kinetics. The resultant mechanism is frequently short-lived because surface chemical kinetics describes the reaction rate in a phenomenological way as a function of the reactant coverage and surface temperature, proposing a consistent reaction mechanism. Most of the mechanisms deduced on ill-defined surfaces along these lines were discarded in the 1960s after re-examination by surface science techniques including vibration and photoelectron spectroscopies on well-defined surfaces [1,2]. This passive status of surface chemical kinetics has not changed even after the examination of many simulations for the description of the inhomogeneous reactivity of surface species from a mean-field approximation [3] or Monte Carlo simulations on lattice gas models [4]. Fortunately, surface 
science has explained the mechanism of the remarkable sensitivity of chemical reactions toward surface structures, for example, in the synthesis of ammonia $\left(\mathrm{N}_{2}+3 \mathrm{H}_{2} \rightarrow 2 \mathrm{NH}_{3}\right)$ on iron surfaces [5] and $\mathrm{CO}$ oxidation $\left(\mathrm{CO}+\mathrm{O}_{2} \rightarrow \mathrm{CO}_{2}\right)$ on platinum metals [6]. Our understanding is, however, still far from that of gas-phase reactions. We still do not have a suitable method for directly obtaining structural information about the reaction site or active intermediates through the reaction itself. In gas-phase reactions, methods for approaching the potential energy surface (PES) have been established using both chemical kinetics and reaction dynamics [7]. This paper reviews energy partitioning requisite for surface reaction dynamics in both reactant adsorption and product desorption. In the former, electronic excitations take place, showing non-adiabatic processes. In the latter, knowledge of this partitioning opens reaction dynamics focusing on spatial and energy distributions of desorbing products, which can deliver structural information of active intermediates and product formation sites.

In general, a chemical reaction must be characterized with respect to not only chemical kinetics but also reaction dynamics because it is always accompanied with energy partitioning. No structural information is provided by chemical kinetics, since the determined reaction rate constants carry only scalar rate parameters, such as the activation energy and a pre-exponential factor. The structural information on surface-reaction sites and the configuration of the transition state will be found in reaction dynamics dealing with energy partitioning as well as the product velocity distribution in terms of the PES, as is usually the case in gas-phase reactions. Information on the transition state conformation (TS) in gas-phase reactions is deduced from the spatial and energy distributions of emitted products before energy dissipation for various collision energies [7] or by femto-second spectroscopy during molecular transformation [8]. In the case of surface reactions, such information, if obtained, will be able to provide the structure of active sites or intermediates which have been surveyed for nearly a century. The active center theory was proposed by Taylor in 1925 [9].

An approach to TS in surface reactions has been partly successful in limited cases and has been useful in the identification of active intermediates and reaction sites [10]. In general, the TS in a surface reaction is still difficult to examine experimentally, even by means of fast surface spectroscopy, because nascent product molecules are quickly thermalized to the surface temperature before detection [11]. Thus, the density functional theory (DFT) with a generalized 
gradient approximation (GGA) has yielded many proposals for TS configurations without experimental verification $[12,13]$. At present, there is no method for determining the energy distributions of nascent products on metal surfaces. Thus, only limited surface reactions, which emit product molecules with hyperthermal energy, have been examined for the configuration analysis of TS.

\section{Potential energy surface and energy partitioning}

Here, reaction dynamics in the gas phase is briefly exemplified for comparison with that in surface reactions. This comparison clarifies what is lacking in surface reaction dynamics. The merit of studying gas-phase bimolecular reactions is that (i) the initial conditions of the reactants can be specified, (ii) there are physical quantities, such as the total momentum, energy, and angular momentum conserved before and after the collision event, (iii) by measuring the velocity distribution of products in each vibrational and rotational state, detailed dynamical information can be deduced, such as differential cross sections (DCS) and flux intensities for each internal state of the product molecules as a function of the relative velocity vector of a product in the so-called center-of-mass (c.m.) system of coordinates, in which the total linear momentum for a pair of products is zero, and (iv) the potential energy surface of a given reaction can be constructed. To construct the PES from the experiment, the DCS or flux intensities must be measured at several collision energies. Of course, this kind of analysis has been performed for several gas-phase reactions.

Here, the analysis of the reaction $\mathrm{F}+\mathrm{H}_{2} \rightarrow \mathrm{HF}+\mathrm{H}$ is briefly summarized as one of the most thoroughly studied systems. This reaction is exothermic by $32.08 \mathrm{kcal} / \mathrm{mol}$. This energy along with the relative translational and internal energy of the reactants is partitioned into the quantum states as well as the relative translational energy of products. The potential energy diagram of the reaction is shown in Fig. 1 for reactant $\mathrm{H}_{2}$ in the rotationally and vibrationally ground state. The energy level for each vibrational state of product HF is also drawn. From the analysis of the infrared chemiluminescence spectrum of nascent product HF [14], the fractional formation ratio for each vibrational level was calculated at $0.31\left(v^{\prime}=1\right): 1.00\left(v^{\prime}=2\right): 0.47\left(v^{\prime}=3\right)$; i.e., most of the HF products are vibrationally excited to the second $\left(v^{\prime}=2\right)$ and third $\left(v^{\prime}=3\right)$ 
vibrational levels. The mean fraction of the total energy available in products entering vibration is $66 \%$ and the remaining energy is converted into the rotational $(8 \%)$ and translational (26\%) modes. On the other hand, using a crossed-molecular beam technique a velocity-selected fluorine atom beam is crossed with a velocity-selected supersonic $\mathrm{H}_{2}$ beam and the product HF molecules emitted from the crossing zone are detected using a rotatable mass spectrometer. The velocity distribution is measured at each scattering angle using a time-of-flight technique. The differential cross sections are deduced from these data [15-17].

The intensity of $\mathrm{HF}$ formed from the reaction $\mathrm{F}+$ para- $\mathrm{H}_{2} \rightarrow \mathrm{HF}+\mathrm{H}$ at a relative collision energy of $1.84 \mathrm{kcal} / \mathrm{mol}$ is plotted in Fig. 2a as a function of the laboratory scattering angle $(\Theta)$ along with a Newton diagram for this system (lower panel), where $\Theta$ is referred from the F-atom beam direction. The HF intensity profile exhibits three peaks. The contributions of vibrationally and rotationally excited HF molecules were assigned by analyzing the velocity distributions. From the conservation of the linear momentum for the whole FHH system, the velocity vector of the c.m. of FHH is directed toward point G from the origin of the Newton diagram at $\Theta=18.2^{\circ}$ (Fig. 2a). Since the forces acting among the atoms involved depend only upon their relative positions, one needs to obtain the dynamical information in the c.m. system.

The circles marked with $v^{\prime}=1, v^{\prime}=2$, and $v^{\prime}=3$ are the endpoints of the relative velocity vectors calculated from the relative translational energy available for the rotationally ground HF products at the $v^{\prime}=1,2$, and 3 states, respectively. A contribution from the $v^{\prime}=3$ state of the HF product is remarkable in a region of small scattering angles. At each laboratory angle $\Theta$, two velocity components are seen; i.e., one corresponding to HF $\left(v^{\prime}=3\right)$, which leaves with the vector sum of the relative HF velocity in the c.m. system and the velocity of the center of mass of F-H-H $\overrightarrow{\alpha \in}$, achieving a larger speed than OG, and the other, which moves more slowly than OG.

Figure $2 \mathrm{~b}$ is the so-called c.m. flux-velocity contour map extracted from the velocity distributions observed at all laboratory scattering angles. This distribution is also called differential cross section (DCS). Actually, the rotational modes are also excited, showing significant intensities between circles of $v^{\prime}=2$ and 3 (Fig. $2 \mathrm{~b}$ ) as well as inside the circle of $v^{\prime}=$ 3. The product is emitted in a three-dimensional space and the flux distribution is symmetric 
around the initial collision velocity vector. The energy content and its flux intensity involved in the product are sensitive to the scattering angle. The following characters can be derived from the figure. The PES constructed in theoretical treatments must involve these characters.

(1) All HF molecules are vibrationally excited. Their population is sensitive to the angle.

(2) Except for $v^{\prime}=3$ products, HF is emitted into the backward direction.

(3) HF with $v^{\prime}=3$ is preferentially scattered into the forward direction.

Comparison of experimental results with the theoretical DCS's and total reaction cross sections calculated on various trial PES's for various initial conditions are expected to yield a suitable PES. Figure 3a shows a PES for a collinear F-H-H configuration and the dashed line represents the minimum energy path (reaction coordinate) for $\mathrm{F}+\mathrm{H}_{2}(0,0)$ reaction [18]. $\mathrm{H}_{2}(0,0)$ stands for $\mathrm{H}_{2}$ in the rotationally and vibrationally ground state. The highest potential energy state (saddle point) appears at an F-H separation of $170 \%$ of that of the H-F bond distance, consistent with formation of a highly vibrationally excited HF [19]. On the other hand, at the transition state the H-H separation is close to that in the ground state. This yields comparatively high recoil energy for $\mathrm{H}+\mathrm{HF}$ pair due to a repulsive force exerted between them. Takayanagi and Sato[20] have attained a PES with a bent transition state configuration (shown in Fig. 3b) which affords the best-fit theoretical DCS's to all of the DCS data [16, 17]. Introduction of this bent conformation is required to reproduce the dominant forward scatterings for $v^{\prime}=3$ state HF [20], in which F-H-H intermediate should turn around by almost $180^{\circ}$.

\section{Energy transfer on metal surfaces}

The reaction dynamics in the gas phase can clearly describe the energy partitioning into each available mode of products because the energy conservation rule holds for each reaction system. On the other hand, the energy flow in a chemical reaction on metal surfaces has several channels with different time constants. Sometimes, the energy flow starts at the point where gaseous reactants are far from collision points because the electronic excitations occur easily on metal surfaces. Furthermore, the initial energy states of adsorbed reactants cannot be 
selected towards its reaction. Except for the Eley-Rideal (ER) type reactions in which the initial state for one of the reactants can be selected using an atom beam [21], reactants on the surface are always thermalized to the surface temperature. Nascent products on the surface are also quickly thermalized before detection. The relaxation time of vibrationally excited molecules is in the order of picoseconds on metals (see Table 1) [22-23]. Eventually, even fast surface spectroscopy yields only chemical kinetics because the observed product species is already thermalized to the surface temperature.

In fact, this fast energy transfer takes place through electronic excitations [24]. Its effect had been ignored in the well-accepted adiabatic assumptions (the Born-Oppenheimer approximation, in which the nuclei appear static toward the electrons, and the electrons then generate a potential that governs the nuclear motion [25]) on metal surfaces. In other words, the electronic state has been assumed to be in the ground state throughout chemical reactions. However, recent experiments clearly show the inadequacy of this assumption. The contribution from the electronic excitation is clear in the energy transfer from the vibrationally excited molecules to the metal surface.

The relaxation time $(\tau)$ of vibrationally excited $\mathrm{CO}$ is about 2 ps on a metal surface (Table 1); i.e., $63 \%$ of the excited $\mathrm{CO}$ loses energy within this time interval $[22,23]$. The $\mathrm{C}-\mathrm{O}$ vibration is repeated about 100 times during this period. The relaxation takes about 3 orders longer on a semi-conductor [26,27]. It is extended 3 orders further on insulator $\mathrm{NaCl}$, close to that in the gas phase $[28,29]$. Such large differences in the relaxation time are due to different mechanisms of the energy flow. On metal surfaces, the electronic excitation (excited electron-hole pair formation) takes place to absorb the released energy once and transfer it to the lattice phonon modes. The $\mathrm{CO}$ vibration energy (about $250 \mathrm{mV}$ ) must be transferred in one event because of the non-divided quantum. This amount must be fully transferred to receivers. The metal lattice phonon (about $10 \mathrm{mV}$ in energy) is too small [30]. This transfer pathway is not fast, since about 25 phonons must be simultaneously excited. On the other hand, the excitation of electronic states is much easier in a metal crystal, since the energy state is continuous in the conduction band. On the other hand, on a semiconductor surface, the electronic excitation is much slower, since the conduction band is mostly occupied. The excitation into the upper conduction band must occur. 


\section{Energy flow in adsorption}

The above electronic excitation in metals can be directly observed in chemical adsorption processes using a Schottky barrier device [31]. The bond formation in chemical adsorption releases energy of a few eV, exciting the electronic states. The lifetime of excited electrons with one $\mathrm{eV}$ above the Fermi level is short, around $30 \mathrm{fs}$, and shows a mean free path of about $30 \mathrm{~nm}$ [32]. Nienhaus constructed a device to detect such electrons excited in chemisorptions; i.e., a thin metal layer is deposited on a Si surface. A potential energy barrier is formed in the interface between the metal and the silicon. Ideally, its height can reach the difference in the work function between the metal and Si. This Schottky barrier is negative, $0.7 \mathrm{eV}$ high, when a silver layer is deposited on an $\mathrm{n}-\mathrm{Si}(111)$ (Fig. 4). The electron that passes this barrier from the metal side cannot return, yielding a negative current between the metal layer and Si. During chemical adsorption of hydrogen atoms, a significant current (chemicurrent) was simply measured on this device. Figure 4 shows the variation of this chemicurrent during $\mathrm{H}$ atom adsorption on Ag films on $\mathrm{n}-\mathrm{Si}(111)$ [31]. The chemicurrent decays exponentially as expected for atom adsorption. The initial chemicurrent yield was estimated to be $4.5 \times 10^{-3}$ electrons per adsorption event. This does not mean a rare event of electronic excitations because of the detection of only parts of excited electrons over the potential barrier. Such a chemicurrent is observed on the adsorption of hydrogen or oxygen atoms on $\mathrm{Mg}$, $\mathrm{Ag}$, and $\mathrm{Au}$ films on $\mathrm{Si}$ [33]. The energy released in the chemical bond formation is first transferred into the electronic excitation before it is transferred into phonon modes. Similar electronic excitations may occur during desorption, but such excitations have not been successfully observed because of the disturbance by heating procedures.

These excited electrons are not emitted out of transition metal surfaces because of the high work function value, 4-5.5 eV [34]. Electrons (called exo-electrons) can be emitted when chemisorption with strong bonding takes place on metal surfaces with low work functions, 1-2 $\mathrm{eV}$ [35]. This exo-electron emission phenomenon was already noticed in 1905 [36]. The phenomenon was reproduced in the 1970s in the Ertl's group using surface science techniques [37]; i.e., a few monolayers of $\mathrm{Cs}$ are deposited on $\mathrm{Ru}(0001)$ and exposed to $\mathrm{O}_{2}$ at $220 \mathrm{~K}$ (Fig. 
5). The exo-electron appears at around one $\mathrm{L}\left(1\right.$ Langmuir $=1 \times 10^{-6}$ Torr $\left.\mathrm{sec}=1.33 \times 10^{-4} \mathrm{~Pa} \mathrm{sec}\right)$ of $\mathrm{O}_{2}$ exposure and disappears above $2 \mathrm{~L}$ after a steep maximum. Work functions determined by photoelectron spectroscopy are also shown. The value decreases once and increases slowly to the value on $\mathrm{CsO}_{2}$ layers. The exo-electron is observed when the work function decreases to $1.3 \mathrm{eV}$. The oxidation state of Cs was studied by X-ray photoelectron spectroscopy [38]. The formation of $\mathrm{Cs}_{2} \mathrm{O}_{2}$ is completed at the work function minimum. After this level, the formation of $\mathrm{CsO}_{2},\left(\mathrm{Cs}_{2} \mathrm{O}_{2} \rightarrow \mathrm{CsO}_{2}\right)$, begins, and exo-electrons are emitted. However, its emission is suppressed since the resultant oxide has a work function above $2 \mathrm{eV}$. Similar exo-electron emission is observed in the adsorption of halogens on alkaline metal surfaces [39].

In the course of this oxygen (or halogen) exposure, negative oxygen (or halogen) ions are also emitted. It was proposed that a metal electron transfers into $\mathrm{O}_{2}$ (or $\mathrm{Br}_{2}$ for example) near the surface before adsorption and the resultant ion $\mathrm{O}_{2}^{-}\left(\mathrm{or}_{2}{ }^{-}\right)$is accelerated on the surface, yielding the emission of $\mathrm{O}^{-}\left(\mathrm{or} \mathrm{Br}^{-}\right)$and electrons. According to energy analysis, the electron was proposed to be emitted through an Auger process of a positive hole created by the impact of negative $\mathrm{O}_{2}{ }^{-}[40]$. A similar mechanism with intermediate negative-ion formation resulting from electron transfer from metal was also proposed in $\mathrm{O}_{2}$ dissociation on $\mathrm{Al}(111)$ [41] and scattering of vibrationally excited $\mathrm{NO}$ on Cs-covered $\mathrm{Au}(111)[42,43]$.

\section{Structure-informative desorption}

From some thermally activated surface reactions, product molecules with hyperthermal energy are emitted into a three-dimensional space, showing the energy content dependent on the desorption angle [44]. Their flux and energy distributions must involve the structure information of reaction sites in a way similar to those in gas-phase reactions. The distributions, however, must be referred to as both the surface crystal azimuth and desorption (polar) angles, instead of the relative collision velocity vector of one reactant in the gas phase reaction; i.e., surface-structural information is provided from the crystal azimuth dependence of the flux and energy [45]. No surface-structural information can be obtained when the flux distribution is symmetric about the surface-normal axis or no azimuth dependence is found in the energy in either internal or translational modes. For example, structural information of reaction sites is 
missing in the energetics of $\mathrm{H}_{2}$ desorption in thermally activated $2 \mathrm{H}(\mathrm{a}) \rightarrow \mathrm{H}_{2}(\mathrm{~g})$ on metal surfaces [46]. No azimuth-angle dependence has been found in the flux of desorbing hydrogen molecules $[47,48]$.

At present, only four thermally activated reactive desorption processes, $\mathrm{CO}(\mathrm{a})+$ $\mathrm{O}(\mathrm{a}) \rightarrow \mathrm{CO}_{2}(\mathrm{~g})$ [49], $\mathrm{C}(\mathrm{a})+\mathrm{O}(\mathrm{a}) \rightarrow \mathrm{CO}(\mathrm{g})$ [50], $\mathrm{N}_{2} \mathrm{O}(\mathrm{a}) \rightarrow \mathrm{N}_{2}(\mathrm{~g})+\mathrm{O}(\mathrm{a})$ [51], and $2 \mathrm{~N}(\mathrm{a}) \rightarrow \mathrm{N}_{2}(\mathrm{~g})$ [52], have exhibited remarkable desorption- and azimuth-angle dependences of the flux intensity and/or the energy of products. Such information can provide the potential for structural information of the reaction sites as well as active surface species at the transition state. It should be noted that the resultant structural information depends on the desorption mechanism, dissociative or associative [10]. Desorbing products with hyperthermal energy have experienced repulsive interactions from counter parts. In dissociative desorption, the direction of this repulsive force is apparent along the ruptured bond axis. On the other hand, in associative desorption, the repulsive forces exerted from reaction sites are significant, since several bonds are involved. Fortunately, this concern provides a method for site assignment.

The above classification is reminiscent of two popular mechanisms in electron-stimulated desorption ion angular distribution (ESDIAD). In this method, emitted ions or neutral fragments are analyzed in an angle-resolved way when their emission is initiated by the injection of electrons, photons or ions [53-55]. For the excitation leading to dissociations, the Menzel-Gomer-Redhead (MGR) model has frequently been used [56-58]; i.e., the adsorbed system in a ground-state configuration is converted to some excited states (antibonding, metastable or ionic in nature). These excited states normally involve valence-level excitations and emit fragments with high kinetic energy during their subsequent dissociation because the electronic excitation occurs so swiftly that nuclear motion is negligible and then the fragment receives high repulsive forces from the counterpart. The fragment is considered to be emitted mostly along the ruptured bond axis, and their spatial distributions then yield the orientation of parent molecules. Indeed, this approach to the orientation of adsorbed molecules is available only for limited emission far from the surface-parallel direction (for example, less than about 50 degrees to the surface normal [54]) because of the disturbance toward the fragment trajectory from the surface itself through image charge interactions, neutralization or scattering.

In the thermal dissociation of adsorbed molecules, however, the above direct fragment 
emission along the ruptured bond axis has never been confirmed. In thermal decomposition, the released fragment is quickly thermalized to the surface temperature, and the eventual desorption is mostly in an associative manner. Thus, the recently reported inclined $\mathrm{N}_{2}$ emission is exceptional, showing similarity to MGR-type emission. It is initiated by $\mathrm{N}_{2}$ emission from the thermal $\mathrm{N}_{2} \mathrm{O}$ decomposition. The inclined $\mathrm{N}_{2}$ emission is found on $\operatorname{Pd}(110), \operatorname{Rh}(110), \operatorname{Ir}(110)$, and $\mathrm{Rh}(100)$ [10]. In this thermal dissociation, both the terminal oxygen and nitrogen atoms in adsorbed N-N-O interact with surface atoms, and the resultant fragment $\mathrm{N}_{2}$ is first emitted in a ballistic way along the surface plane as observed in the thermal $\mathrm{O}_{2}$ dissociation on $\operatorname{Pt}(111)$ $[59,60]$, according to molecular dynamic simulations based on the potential energy surface derived from DFT-GGR work [61]. This surface-parallel emission is, in fact, difficult to observe directly because of scattering by either surface atoms or coadsorbed species. The fragment $\mathrm{N}_{2}$ is eventually emitted into an inclined way in the plane along the $\mathrm{N}_{2} \mathrm{O}$ bond axis. Thus, the orientation of parent molecules is preserved in the resultant fragment distribution only in a two-dimensional way.

On the other hand, the emission in electron-simulated desorption is mostly collimated along the surface normal on flat surfaces when it is induced from species without chemical bonding to surfaces such as physical adsorption states [62]. In such an extreme case, the species positively ionized by the electron injection is first accelerated toward the surface by image forces and then repulsively desorbed after neutralization (the Antoniewicz mechanism [63]). The repulsive force is highly exerted from the adsorption site because of the absence of directed ruptured bonds. A mechanism close to the latter is operative for the associative desorption such as $\mathrm{CO}(\mathrm{a})+\mathrm{O}(\mathrm{a}) \rightarrow \mathrm{CO}_{2}(\mathrm{~g})$ and is useful for assigning the product formation place as summarized in Chapters 9 and 10. Another mechanism close to the former (the MGR model) works for the dissociative desorption of $\mathrm{N}_{2} \mathrm{O}(\mathrm{a}) \rightarrow \mathrm{N}_{2}(\mathrm{~g})+\mathrm{O}$ (a) and is powerful enough to assign active intermediate-emitting products, as described in detail in Chapters 7 and 8.

Introducing reactants onto surfaces using a molecular beam has advantages only in the measurement of the surface residence time of incident reactants or when the product molecules have some memory of the momentum of incident species. The latter case is found in the reactions with the Eley-Rideal mechanism observed in the interactions of atom beams with surface species [64]. Except for these ER reactions, the incoming reactants are quickly 
thermalized on the surface before reaction. They will participate in a Langmuir-Hinshelwood type reaction. Memory of the incident momentum of reactants is completely lost in desorbing product molecules. This condition simplifies the design criteria of an apparatus for angle-resolved (AR) analysis of desorbing reaction products, which takes care of only desorption events and reaction conditions. The latter can be definitely controlled in various transient experiments, for example, temperature-programmed desorption (TPD) and pressure jump/drop response as well as under steady-state reaction conditions.

\section{Angle-resolved desorption measurements}

In ESDIAD, angle-resolved (AR) desorption measurements can be performed in a single ultrahigh vacuum chamber. With the use of short laser pulses, electron beams or ion beams, the signal due to product species directly moving from the surface to the detector can be distinguished in time-resolved measurements because of the delayed arrival of species scattered by the chamber wall. This can be extended to AR internal energy measurements by using a two-dimensional microchannel plate (MCP) detector [65].

On the other hand, in thermal reactions, at least three chambers must be combined because at least two slits should be operative between the sample surface and the detector in an analyzer. The principle behind the apparatus is drawn in Fig. 6. The apparatus is an ultrahigh vacuum system composed of a reaction chamber, a chopper housing, and the analyzer, which are separately pumped. High-speed pumping is requisite in either the reaction chamber or the chopper house, as numerically analyzed by Kobayashi and Tuzi [66]. In this figure, a very large pumping rate can be established by a copper plate cooled to around $25 \mathrm{~K}$ or a Ti getter. The flux and both the translational and internal energies of desorbing products must be analyzed after these AR procedures. Otherwise, the molecules scattered in the reaction chamber or chopper house penetrate the analyzer and contribute to the AR signal, since only about $0.01 \%$ of the emitted molecules can directly enter the ionization region of the analyzer mass spectrometer at an angle-resolution of 1 degree. The reaction chamber has standard facilities for surface analysis, such as low-energy electron diffraction (LEED) and X-ray photoelectron spectroscopy optics, an ion gun and a quadruple mass spectrometer (QMS). The 
chopper house has a narrow slit facing the reaction chamber and contains a cross-correlation chopper blade for time-of-flight (TOF) measurements (see the inset in Fig. 6)[67]. A sample crystal is rotated on the mass spectrometer ionizer axis to adjust the desorption angle ( $\theta$; polar angle) and the crystal azimuth $(\phi)$. In Fig. 7(a), both angles are defined on $\operatorname{Pd}(110)$, where the azimuth is measured from the [001] direction.

For internal energy measurements after AR procedures, the optics for infrared (IR) chemiluminescence collection or the laser light for resonance-enhanced multiphoton ionization (REMPI) should be focused on the product flow after the second slit. The product density there is reduced to about $0.1 \%$ of that at several $\mathrm{mm}$ from the surface in the non-AR way $[68,69]$. Recently, Yamanaka constructed an apparatus for the IR emission detection of desorbing product $\mathrm{CO}_{2}$ using AR procedures. A meaningful signal was detected after the background emission was reduced to $0.05 \%$ of that without cooled shielding around the optics [70].

For many years, IR chemiluminescence and REMPI have been applied to the molecules just in front of the surface, several mm away from the surface (see the non-AR in Fig. 6). The former was used for product $\mathrm{CO}$ in carbon oxidation or $\mathrm{CO}_{2}$ in $\mathrm{CO}$ oxidation. The latter was for $\mathrm{NO}, \mathrm{H}_{2}$ and $\mathrm{N}_{2}$, in their thermal desorption [68]. All these measurements are in the non-AR way.

\section{Structure-information in dissociative desorption}

In thermal dissociative (repulsive) desorption, the orientation of parent molecules is preserved in the spatial distribution of desorbing fragments in a manner different from that in ESDIAD. Adsorbed $\mathrm{N}_{2} \mathrm{O}$ dissociates even below $100 \mathrm{~K}$ on open surfaces, such as the (110) plane of $\mathrm{Ni}$, $\mathrm{Pd}, \mathrm{Rh}$ and $\mathrm{Ir}, \mathrm{Rh}(100)$ and $\mathrm{Ni}(100)$ [10,71]. Inclined $\mathrm{N}_{2}$ emission is observed in the heating of $\mathrm{N}_{2} \mathrm{O}$-covered surfaces or in the exposure of $\mathrm{N}_{2} \mathrm{O}$ to a clean surface at a fixed temperature as well as in its steady-state reduction in the presence of reducing reagent. The typical AR-TPD spectra of desorbing $\mathrm{N}_{2}$ during heating of $\mathrm{N}_{2} \mathrm{O}$-covered $\mathrm{Pd}(110)$ are reproduced at small $\mathrm{N}_{2} \mathrm{O}$ coverage in Fig. 8 [72]. Here, a clean palladium surface was exposed to $\mathrm{N}_{2} \mathrm{O}$ at around $95 \mathrm{~K}$, and, in the subsequent heating, $\mathrm{N}_{2}$ desorption was monitored in an AR way. The spectrum shape is sensitive to the desorption angle (Fig. 8a-b). It shows four peaks, $\beta_{1}-\mathrm{N}_{2}(150 \mathrm{~K})$, 
$\beta_{2}-\mathrm{N}_{2}(134 \mathrm{~K}), \beta_{3}-\mathrm{N}_{2}(123 \mathrm{~K})$ and $\beta_{4}-\mathrm{N}_{2}(110 \mathrm{~K})$. The first peak is found at high $\mathrm{N}_{2} \mathrm{O}$ exposures, and the last, at low exposures. $\beta_{2}-\mathrm{N}_{2}$ yields a cosine distribution, indicating the desorption of adsorbed $\mathrm{N}_{2}$. In fact, $\mathrm{N}_{2}$ desorption peaks at $134 \mathrm{~K}$ when $\mathrm{N}_{2}$-covered $\mathrm{Pd}(110)$ is heated. On the other hand, $\beta_{1}-\mathrm{N}_{2}, \beta_{3}-\mathrm{N}_{2}$ and $\beta_{4}-\mathrm{N}_{2}$ commonly reveal inclined emission at $43-50^{\circ}$ off the surface normal toward either the [001] or [001] direction (Fig. 8c-e). Such emission is due to the decomposition of $\mathrm{N}_{2} \mathrm{O}$ oriented in the [001] direction. The formation of each peak is due to the enhanced activation energy of $\mathrm{N}_{2} \mathrm{O}$ dissociation modified by deposited oxygen [72,73]; i.e., the adsorption heat of $\mathrm{N}_{2} \mathrm{O}$ is largely increased in the presence of adsorbed oxygen in a small amount [74]. Surface oxygen deposited before or during the subsequent heating can yield sites with different activation energies depending on the extent of oxygen coordination [75.76].

The fragment $\mathrm{N}_{2}$ emission is highly concentrated in the plane along the [001] direction [52]. A three-dimensional distribution of desorbing $\mathrm{N}_{2}$ was constructed in the steady-state $\mathrm{N}_{2} \mathrm{O}+\mathrm{CO}$ on $\mathrm{Pd}(110)$ from its angular distributions in the plane along different crystal azimuths (Fig. 9). This reaction is stable on $\mathrm{Pd}(110)$ in a wide temperature range to $800 \mathrm{~K}$ [77]. The azimuth angle $(\phi)$ is defined as a deviation from the [001] direction. Here, a new angle system is necessary for presenting the distribution in three-dimensional ways using the power functions of the cosine of the desorption angles $(\alpha, \beta)$ in different planes (Fig. 7b). According to the rotation defining Eulerian angles, the relation between angles $(\alpha, \beta)$ and $(\theta, \phi)$ is given by $\cos \theta$ $=\cos \alpha \cos \beta$ and $\tan \phi=-\tan \beta / \sin \alpha$ [78]. The experimental AR signals at definite $(\theta, \phi)$ values were converted into the signal intensity at new coordinates $(\alpha, \beta)$. The resultant spatial distribution is well described as $\cos ^{28}(\alpha \pm 45) \cos ^{17}(\beta)$ at $520 \mathrm{~K}$. This distribution is very sharp against the crystal azimuth (Fig. 9b). It supports the idea that $\mathrm{N}_{2} \mathrm{O}$ decomposes after being oriented along the [001] direction in the wide temperature range of 100-800 K.

Adsorbed $\mathrm{N}_{2} \mathrm{O}$ was observed to be oriented along the [001] direction on $\operatorname{Pd}(110)$ by scanning-tunneling microscopy (STM) at $14 \mathrm{~K}$ [78] and by near-edge X-ray-absorption fine structure (NEXAFS) at $60 \mathrm{~K}$ [80]. The co-existing $\mathrm{N}_{2} \mathrm{O}$ in an upright form bonding through the terminal nitrogen [81] is not reactive. According to DFT-GGA calculations carried out by Kokaji, the nascent $\mathrm{N}_{2}$, immediately after dissociation of the N-O bond, is attractive toward the nearest metal atom on $\operatorname{Pd}(110)$ and $\operatorname{Rh}(110)$ and repulsive toward the counter product oxygen; i.e., the bond of the terminal nitrogen to the metal atom is not broken at the dissociation 
moment of the N-O bond $[61,82,83]$. Thus, the product $\mathrm{N}_{2}$ has been proposed to be first emitted parallel to the surface plane and then scattered with deposited oxygen. On the other hand, the collimation angle around $45^{\circ}$ on $\operatorname{Pd}(110)$ is not shifted either under AR-TPD conditions with deposited oxygen and remaining $\mathrm{N}_{2} \mathrm{O}$ or in the steady-state $\mathrm{N}_{2} \mathrm{O}+\mathrm{CO}$ reaction under both $P_{\mathrm{N} 20 . \mathrm{C}}>P_{\mathrm{CO}}$ and $P_{\mathrm{N} 2 \mathrm{O} . \mathrm{C}}<P_{\mathrm{CO}}$ conditions, where $P_{\mathrm{N} 20 . \mathrm{C}}$ is the kinetic critical pressure of $\mathrm{N}_{2} \mathrm{O}$ [77]. In the former of $P_{\mathrm{N} 2 \mathrm{O} . \mathrm{C}}>P_{\mathrm{CO}}$, the surface is partly covered by oxygen, and, in the latter, the surface is covered by $\mathrm{CO}$. The surface is essentially clean at temperatures higher than $600 \mathrm{~K}$ when $P_{\mathrm{N} 2 \mathrm{O} . \mathrm{C}}<P_{\mathrm{CO}}$. The collimation angle on $\operatorname{Pd}(110)$ is insensitive to co-adsorbed species, different from that on $\mathrm{Rh}(110)$.

Nearly surface-parallel $\mathrm{N}_{2}$ emission can be observed on $\mathrm{Rh}(110)$ at around $300 \mathrm{~K}$ when deposited oxygen is removed by hydrogen [84]. The $\mathrm{N}_{2}$ emission is collimated at around $80^{\circ}$ off normal. This parallel emission is not largely disturbed by adsorbed species because of either the absence of hydrogen atoms at high temperatures $[85,86]$ or their small scattering cross section [10]. In the steady-state $\mathrm{N}_{2} \mathrm{O}+\mathrm{CO}$ reaction, the collimation angle shifts from $65^{\circ}$ off normal towards $45^{\circ}$ with decreasing surface temperature in the range of 530-450 $\mathrm{K}$ [87]. This shift is due to increasing amounts of adsorbed $\mathrm{CO}$. $\mathrm{CO}$ (a) retards the $\mathrm{N}_{2} \mathrm{O}$ adsorption and also shifts the collimation angle of $\mathrm{N}_{2}$ emission. The latter effect is due to the scattering of nascent $\mathrm{N}_{2}$ moving parallel to the surface plane because of the large scattering cross section of standing CO. Adsorbed CO suppresses the component that is closely surface parallel. The remaining component is collimated at around $45^{\circ}$. Thus, there must be another mechanism to yield the collimation angle around $45^{\circ}$ regardless of the co-adsorbed species.

The $\mathrm{N}_{2}$ desorption is highly concentrated in the plane along the ruptured bond axis. On $\operatorname{Pd}(110)$, the $\mathrm{N}_{2}$ emission is sharply distributed in a narrow range of the azimuth angle (Fig. 9b). In order to leave the surface with a collimation angle of around $45^{\circ}$, the nascent $\mathrm{N}_{2}$ must receive repulsive forces from metal atoms as well as the repulsive force exerted from the counter-product oxygen. In the former repulsion, the force must be induced from either the translational or the rotational mode. According to Kokaji's molecular dynamics (MD) simulations [61], the rotational motion in the nascent $\mathrm{N}_{2}$ is highly excited in a cartwheel form because the adsorbed $\mathrm{N}_{2} \mathrm{O}$ is bent and the nascent fragment will receive strong torque. In the subsequent energy partitioning, the rotational energy might be used to enhance the momentum 
along the surface normal. The AR internal and translational energy distributions of $\mathrm{N}_{2}$ products, if obtained using state specific detection techniques, would facilitate the understanding of the above energy partitioning in terms of the PES and the topology of the reaction site.

\section{8. $\quad \mathbf{N}_{2}$ emission in NO reduction}

Nitrous oxide was frequently proposed to be an intermediate in NO reduction on metal catalysts. In fact, $\mathrm{N}_{2} \mathrm{O}$ is, to some extent, always found in $\mathrm{NO}$ reduction over metal catalysts [88]. It is generally accepted that, at high temperatures, the surface-nitrogen removal proceeds as follows: $2 \mathrm{~N}(\mathrm{a}) \rightarrow \mathrm{N}_{2}$ (g) $\left(\beta-\mathrm{N}_{2}\right.$ formation). On the other hand, at temperatures below $500-700$ $\mathrm{K}$, the $\delta-\mathrm{N}_{2}$ formation via $\mathrm{N}(\mathrm{a})+\mathrm{NO}(\mathrm{a}) \rightarrow \mathrm{N}_{2} \mathrm{O}(\mathrm{a}) \rightarrow \mathrm{N}_{2}(\mathrm{~g})+\mathrm{O}(\mathrm{a})$ was suggested to contribute to the $\mathrm{N}_{2}$ emission [89]. It has been difficult to find direct evidence of the participation of this species toward $\mathrm{N}_{2}$ formation because of the absence of spectroscopic evidence of adsorbed $\mathrm{N}_{2} \mathrm{O}$ in the course of catalyzed NO reduction [90,91].

AR product desorption measurements in the steady-state reaction of NO reduction, however, show the inclined $\mathrm{N}_{2}$ desorption characterizing the $\mathrm{N}_{2} \mathrm{O}$ decomposition under limited conditions. This is not unreasonable because adsorbed $\mathrm{N}_{2} \mathrm{O}$ can be oriented in a suitable way before dissociation even at $800 \mathrm{~K}$. A surface residence time of approximately nanosecond order is long enough to be oriented along the [001] direction. Structure-sensitive desorption dynamics provides the most direct method for identifying short-lived intermediates. On the other hand, such short surface residence is not long enough to allow detection of the species by means of surface vibration and photoelectron spectroscopies. With surface spectroscopy, it is difficult to detect species below $0.01 \%$ of a monolayer under steady-state conditions.

The inclined $\mathrm{N}_{2}$ emission was first found in AR-TPD work of NO-covered Pd(110) by Ikai and Tanaka $[92,93]$. They also confirmed that, by means of an isotope tracer, this inclined $\mathrm{N}_{2}$ comes from the reaction of adsorbed $\mathrm{NO}$ with an adsorbed nitrogen atom; i.e., ${ }^{15} \mathrm{~N}$ atoms are first deposited and further exposed to ${ }^{14} \mathrm{NO}$, and, in the subsequent heating, ${ }^{14} \mathrm{~N}^{15} \mathrm{~N}$ is desorbed into an inclined way, and ${ }^{15} \mathrm{~N}_{2}$ is emitted along the surface normal.

In the $\mathrm{NO}$ reduction on $\mathrm{Pd}(110), \mathrm{Rh}(110)$, and $\mathrm{Rh}(100)$ surfaces, at least three surface-nitrogen removal pathways are operative. The $\beta-\mathrm{N}_{2}$ formation involving $\mathrm{NO}(\mathrm{a}) \rightarrow$ 
$\mathrm{N}(\mathrm{a})+\mathrm{O}$ (a) and $2 \mathrm{~N}(\mathrm{a}) \rightarrow \mathrm{N}_{2}$ (g) is operative at temperatures above $750 \mathrm{~K}$ on $\mathrm{Rh}(100)$, as shown in Fig. 10. The kinetic analysis of this process has shown high activation energy $[94,95]$. The reaction rate increases steeply at around $800 \mathrm{~K}$ and emits $\mathrm{N}_{2}$ along the surface normal. These findings agree well with the kinetics of $\beta-\mathrm{N}_{2}$ formation. On the other hand, the reaction shows small activation energy in the range of $500-800 \mathrm{~K}$. The $\mathrm{N}_{2}$ formation from $\mathrm{NO}(\mathrm{a})+\mathrm{N}(\mathrm{a})$ has been kinetically assumed below about $750 \mathrm{~K}$. In fact, the product $\mathrm{N}_{2}$ is mostly emitted into the inclined way in the plane along either the [001] or [010] direction. The collimation angle is close to $70^{\circ}$, showing $\mathrm{N}_{2} \mathrm{O}$ decomposition. This collimation of desorbing $\mathrm{N}_{2}$ is also observed in AR-TPD of $\mathrm{N}_{2} \mathrm{O}$-covered $\mathrm{Rh}(100)$ surfaces [96]. The spatial distribution work clearly shows that the reaction passes through the intermediate $\mathrm{N}_{2} \mathrm{O}(\mathrm{a})$. A similar pathway shift with increasing surface temperature was derived on $\operatorname{Rh}(110)$ and $\operatorname{Pd}(110)$, where the $\delta-\mathrm{N}_{2}$ formation pathway highly contributes below $500 \mathrm{~K}[77,97]$.

\section{Structures in Associative desorption}

In the above dissociative desorption, the momentum initially given on the fragment is eventually preserved in a two-dimensional way in the spatial distribution of desorbing products. The orientation of parent molecules is derived, yielding information that is useful for identifying short-lived intermediates. On the other hand, in a thermally activated associative desorption, such as $2 \mathrm{~N}(\mathrm{a}) \rightarrow \mathrm{N}_{2}(\mathrm{~g}), \mathrm{C}(\mathrm{a})+\mathrm{O}(\mathrm{a}) \rightarrow \mathrm{CO}(\mathrm{g})$ and $\mathrm{CO}(\mathrm{a})+\mathrm{O}(\mathrm{a}) \rightarrow \mathrm{CO}_{2}(\mathrm{~g})$, the product emission is, to some extent, sharply collimated along the normal direction on flat surfaces $[44,98]$. On stepped surfaces with inclined terraces, the $\mathrm{CO}$ and $\mathrm{CO}_{2}$ desorption is collimated off-normal into inclined ways, showing that the collimation angle is largely controlled by the local shape of the reaction site [45]. From only the spatial and velocity distributions, it is difficult to assign uniquely the ruptured bond controlling the direction of repulsive forces operative toward leaving products. Without internal energy analysis as a function of the desorption angle, the operation of these repulsive forces toward the leaving molecule was treated in a flat barrier model or a way similar to that in the Antoniewicz model in ESDIAD [63].

Here, it is interesting to examine the extent to which the repulsive force exerted from the 
surface site is converted into the translational mode. The well-known Willigen's model assumes the full conversion of the repulsive force into the translational mode [99]. Assuming a flat activation energy barrier for adsorption and the detailed balance principle (or microscopic reversibility), Willigen derived the relation for the angular distribution of desorbing hydrogen molecules from adsorption dynamics; i.e., in dissociative adsorption, incident molecules with energy surmounting a threshold energy barrier can adsorb, and in the reverse process, desorbing molecules hold kinetic energy corresponding to the value above the adsorption barrier height. The resultant angular distribution is described by only the ratio of the activation barrier height for adsorption to the thermal energy, and the total kinetic energy of desorbing molecules increases steeply with increasing desorption angle [10].

Indeed, the translational energy of desorbing molecules in collimated associative emission is usually maximized at the surface normal on a flat surface or remains constant with increasing shift from the surface normal [44]. This discrepancy has been at long last solved by measuring the rotational energy as a function of the desorption angle. Both the rotational and vibrational modes are, to some extent, excited in desorbing products $\mathrm{H}_{2}, \mathrm{~N}_{2}, \mathrm{CO}$ and $\mathrm{CO}_{2}$ in their associative desorption, but their angle dependence has been scarcely reported [68]. Recently, Yamanaka succeeded in observing infrared chemiluminescence from AR product $\mathrm{CO}_{2}$ in $\mathrm{CO}$ oxidation on $\mathrm{Pd}(111)$ (Fig. 11) [70,100]. With increasing desorption angle, the obtained rotational temperature increases steeply; i.e., it is about half of the surface temperature in the normal direction and increases to about twice the surface temperature even at $30^{\circ}$. The vibrational temperature of bending and symmetric stretching modes decreases slowly and the other (anti-symmetric) vibration energy remains fairly constant [101]. On this surface, the translational temperature of $\mathrm{CO}_{2}$ desorbing along the surface normal is high, about $2,300 \mathrm{~K}$ at $T_{\mathrm{S}}=700 \mathrm{~K}$, and decreases close to the surface value at large desorption angles [102]. These observations indicate the occurrence of energy partitioning into either the translation, rotation, or vibration modes in the repulsive desorption event.

The repulsive force is due to Pauli repulsion, since no chemical bond is formed from leaving $\mathrm{CO}_{2}$ to surface metal atoms; i.e., the nascent product is more closely formed to the surface than the equilibrium position of its physical adsorption of bulky $\mathrm{CO}_{2}$ because the reactant oxygen adatom and $\mathrm{CO}$ are in chemical adsorption states before the reaction. Strong 
repulsive forces are induced at the moment of formation between the nascent $\mathrm{CO}_{2}$ and its reaction site. This repulsion decreases steeply with increasing distance from the surface, as expected from the repulsive part in the Morse potential describing a physical adsorption system [103]. In such a steeply decreasing force field, the leaving molecule receives torque in a desorption event when it is inclined against the surface plane (Channel I/rotational excitation); for example, the final bond breaking in the inclined TS toward O-C-O takes place between the reacting adsorbed oxygen and the surface [100]. The resultant desorbing $\mathrm{CO}_{2}$ molecule has a small translational energy, yielding a broad angular distribution, and the rotational modes are excited. The excitation of the vibrational modes depends primarily on the TS configuration and is not necessarily induced in the repulsive desorption event itself. On the other hand, the repulsive force is efficiently converted into the translational mode when the TS is upright or parallel to the surface plane (Channel II/translational excitation)). For example, the final bond breaking takes place between the reacting oxygen atom in the upright TS and the surface or between the center carbon atom in the parallel TS and the surface. The translational mode is excited, yielding a sharp angular distribution, and the rotational modes are not excited. Thus, the product $\mathrm{CO}_{2}$ from both channels predicts the observed angle dependences of translational and internal energies; i.e., the transition state of $\mathrm{CO}_{2}$ on $\mathrm{Pd}(111)$ may partly involve inclined components that are induced by either restricted translations or bending motions. A large contribution of the latter (Channel II) explains the sharp $\mathrm{CO}_{2}$ distribution along the site normal as well as the angle-dependences of both internal and translational energies. The contribution from the former (Channel I) becomes predominant at large desorption angles and yields small translational and high rotational temperatures.

The inclination of the transition state may depend on the crystal azimuth over an anisotropic surface. Thus, this azimuth dependence will appear in both the rotational and translational energies. In fact, Yamanaka succeeded in observing such anisotropy in the internal modes of desorbing $\mathrm{CO}_{2}$ in the $\mathrm{CO}$ oxidation on $\operatorname{Pd}(110)[100,104,105]$. On $\operatorname{Pd}(110)(1 \times 1)$, the rotational temperature in the normal direction, which reaches around $950 \mathrm{~K}$ at the surface temperature of $700 \mathrm{~K}$, decreases quickly with increasing shift from the surface normal in the plane along the [001] direction, whereas it is fairly constant in the plane perpendicular to it; i.e., the rotational mode is excited in $\mathrm{CO}_{2}$ molecules desorbing into the plane along the [1ㅜㅣ] 
direction. A remarkable anisotropy was already observed in the translational modes of desorbing $\mathrm{CO}_{2}$ on this surface under TPD conditions [45]; i.e., the conversion into the translational motion is more efficient in the plane along the [110] direction than in that perpendicular to it (along the [001] direction). The motion of the transition state $\mathrm{CO}_{2}$ along the [001] direction was proposed to be more hindered by large corrugations or easier along the $[1 \overline{1}$ $0]$ direction because of the smooth close-packed arrangements. The above remarkable anisotropy of rotational energy shows now a significant contribution of the energy-partitioning effect. The $\mathrm{CO}_{2}$ molecule in the transition state is suggested to be inclined in the plane along the $[1 \overline{10}]$ direction. The anisotropy in both the rotational and vibrational energies is different when the surface is reconstructed [104,105]. Under the steady-state CO oxidation, the surface structure of $\operatorname{Pd}(110)$ depends on the reaction conditions. It is in a non-reconstructed $(1 \times 1)$ form at a high surface temperature of $700 \mathrm{~K}$ when the $\mathrm{CO}$ pressure is higher than that of $\mathrm{O}_{2}$, since the reconstructed $(1 \times 2)$ form stabilized by oxygen is converted into the $(1 \times 1)$ form above 355 $\mathrm{K}$ by the oxygen removal $[106,107]$. On the other hand, it is reconstructed under oxidative conditions. The angular distribution of desorbing $\mathrm{CO}_{2}$ in the steady-state $\mathrm{CO}$ oxidation on $\mathrm{Pd}(110)$ shows complex behavior [108,109]. Below about $1 \times 10^{-3} \mathrm{~Pa}$ of the $\mathrm{O}_{2}$ pressure, it shows a sharp change at the kinetic critical $\mathrm{CO} / \mathrm{O}_{2}$ pressure ratio where the rate-limiting step switches from $\mathrm{CO}$ adsorption to oxygen dissociation. With increasing $\mathrm{CO}$ pressure, the angular distribution in the plane along the [001] direction changes from the normally directed form into a broad (cosine) one, and the translational temperature also drops to the surface value [108]. On the other hand, above about $1 \times 10^{-3} \mathrm{~Pa}$ of the $\mathrm{O}_{2}$ pressure, the translational temperature does not drop to the surface value even above the critical CO pressure [109]. At high CO pressures and surface temperatures, the surface oxygen seems to be quickly removed to recover the $(1 \times 1)$ form.

The above energy partitioning in the repulsive desorption event is quite similar to that in gas-phase reactions if the inclined form is replaced with the bent one; i.e., the repulsive force is effectively converted into the rotation mode of the fragment when it is operative outward from the gravity center [19]. On the other hand, the force is highly converted into the translational mode when the force is operative toward the gravity center; i.e., in the linear TS in the gas 
phase and in upright or surface-parallel forms on surfaces. The energy partitioning in a repulsive desorption event was once examined in ESDIAD on both experimental and theoretical sides after REMPI was combined with desorption induced by electronic transitions (DIET) $[65,110,111]$. Simple classical trajectory calculations were examined by considering strong repulsive forces converted into the translation, vibration, and rotation modes of desorbing molecules [112]. The results predicted an efficient energy flow into both the translation and vibration modes and a non-efficient one into the rotational modes, consistent with experimental work $[111,113,114]$. In fact, no observable variations are in the angular distribution or azimuth dependence with rotational or vibrational energy of desorbing $\mathrm{CO}$ or NO in DIET on Pt(111).

\section{Normally directed desorption and site switching}

The above energy partitioning in associative (repulsive) desorption gives a base to approach the shape of the reaction site preserved in sharply collimated product emission; i.e., the slope of $\mathrm{CO}_{2}$ formation sites must be memorized in the collimation angle. On flat surfaces, such as $\operatorname{Pd}(111)$, $\mathrm{Pt}(111), \mathrm{Rh}(111)$, and $\mathrm{Pd}(100)$, the product $\mathrm{CO}_{2}$ desorption is sharply collimated along the surface normal, yielding a symmetric distribution about the surface-normal axis $[45,98]$. It indicates a major contribution from Channel II for energy partition in reactive $\mathrm{CO}_{2}$ desorption; i.e., the collimated desorption along the site normal. The $\mathrm{CO}_{2}$ formation takes place on the oxygen adsorption site or nearby even if its activation of the oxygen-metal bond shifts the adsorption position to some extent [115], since the strong oxygen-metal bond must be excited to be broken and the two-, three- or four-fold hollow site suitable for oxygen adsorption on these surfaces is parallel to the bulk surface plane. On the other hand, $\mathrm{CO}_{2}$ desorption is collimated closely along the local normal of the (111) facets on $\operatorname{Pt}(113)(1 \times 2)=[(\mathrm{s}) 3(111) \times 3(001)]$, $\operatorname{Pt}(112)=[(\mathrm{s}) 3(111) \times(001)]$, and $\operatorname{Pt}(335)=[(\mathrm{s}) 4(111) \times(001)]$ as well as the reconstructed $(110)(1 \times 2)$ of $\mathrm{Pt}, \mathrm{Rh}$ and Ir showing $[(\mathrm{s}) 3(111) \times 3(\overline{11})]$. More exactly, the collimation angles of desorbing $\mathrm{CO}_{2}$ on stepped surfaces somewhat deviate toward the surface normal from the local normal of inclined terraces [98]. This deviation completely disappears for the $\mathrm{CO}_{2}$ product with high translational energy, which is induced on $\mathrm{CO}$ - and $\mathrm{O}_{2}$-covered stepped surfaces by 
ultra-violet light $[116,117]$. The corrugation of the repulsive potential toward the thermal reaction product $\mathrm{CO}_{2}$ is less than that of their geometrical shape because of the smaller translational energy. The product $\mathrm{CO}_{2}$ in a $193 \mathrm{~nm}$ photon-induced reaction has high translational energy, about twice that in the thermal reaction, and receives repulsion, showing its potential close to the atomic arrangement. The above stepped surfaces commonly provide inclined facets with a (111) structure wider than two-atom spacing. These (111) facets have adsorption sites available for oxygen with a relatively small binding energy because of the close-packed arrangements. Their reactivity is higher than that on opened (100) planes or step sites. Mobile CO ad-molecules can visit oxygen adatoms in a wide area and react with more active oxygen. Thus, $\mathrm{CO}_{2}$ is formed on either the oxygen adsorption site on (111) planes or nearby. Facets with two-atom spacing are not wide enough to emit the product $\mathrm{CO}_{2}$ in an inclined way. On non-reconstructed $(110)(1 \times 1)$ of $\mathrm{Pd}, \mathrm{Rh}, \mathrm{Pt}$, and $\mathrm{Ir}, \mathrm{CO}_{2}$ desorption is collimated along the surface normal, and its distribution is sharp in the plane along the [001] direction and broad in the plane perpendicular to it.

The above site-normal directed $\mathrm{CO}_{2}$ desorption can be applied to identify $\mathrm{CO}_{2}$ formation sites in the course of the catalyzed reaction. In the steady-state CO oxidation on $\operatorname{Pt}(113)(1 \times 2)$, $\mathrm{CO}_{2}$ desorption mostly collimates along the local normal of (111) facets at low $\mathrm{CO}$ pressures $\left(P_{\mathrm{CO}}<<P_{\mathrm{O} 2}\right)$, where $\mathrm{O}(\mathrm{a})>>\mathrm{CO}$ (a) [118]. On the other hand, its desorption shifts primarily along the (001) facet normal when the CO pressure is close to the kinetic transition point or above it, where $\mathrm{CO}(\mathrm{a})>>\mathrm{O}(\mathrm{a})$. This is reasonable because of the higher reactivity of oxygen on (111) terraces toward adsorbed $\mathrm{CO}$. The oxygen on (001) facets can react with $\mathrm{CO}$ after most of the oxygen is consumed on (111) parts.

The distribution of desorbing $\mathrm{CO}_{2}$ varies when the surface structure changes [119]. For example, reconstructed $\mathrm{Pt}(110)(1 \times 2)$ having three-atom wide terraces is converted into non-reconstructed $(1 \times 1)$ when the CO pressure exceeds a critical kinetic value at which the rate-determining step is switched from $\mathrm{CO}$ adsorption to oxygen dissociation. Above the critical pressure, the amount of adsorbed $\mathrm{CO}$ reaches the level equilibrium to the $\mathrm{CO}$ partial pressure. Concomitantly, the $\mathrm{CO}_{2}$ desorption shifts from the two-directional distribution into the normally directed one whenever the CO coverage reaches half a monolayer or above (Fig. 12). The completed conversion into $(1 \times 1)$ requires adsorbed $\mathrm{CO}$ in the amount of half a 
monolayer. In the former, the $\mathrm{CO}_{2}$ desorption is collimated at about $\pm 26^{\circ}$ off normal, closely along the local normal of the (111) facet. It is interesting to see the two-directional desorption even above the critical point when the CO coverage is below half a monolayer; i.e., the surface is partly covered by $(1 \times 1)$ and $(1 \times 2)$ forms as designated by a "mixed region" in Fig. 12 . The remaining $(1 \times 2)$ reconstructed part still plays the main role in producing $\mathrm{CO}_{2}$, suggesting a high potential of reaction sites on inclined (111) facets. The $\mathrm{CO}_{2}$ formation process itself is very fast and not rate-determining even on reduced active $(1 \times 2)$ areas. The total $\mathrm{CO}_{2}$ formation rate is controlled by the supply of oxygen atoms to the reaction place.

\section{Summary and future work}

In studies of the above-described associative $\mathrm{CO}_{2}$ or dissociative $\mathrm{N}_{2}$ desorption, the distributions of the flux and both translational and internal energies of desorbing products with hyperthermal energy have provided structural information about its reaction site; i.e., the site shape for associative desorption, as well as the orientation of parent molecules for dissociative reactions. The resultant desorption dynamics provides the most direct site-identification method applicable in the course of catalyzed reactions. Little is known about the energy transfer between surfaces and desorbing molecules. Electronic excitations take place in adsorption-bond formation of chemical species with metal surfaces. The adsorption energy is not directly transferred into phonon modes. Similar situations may be expected regarding the breakage of chemical adsorption bonds.

For surface chemical reactions, the state-resolved analysis is limited to a few desorption processes. We still do not have a method for analyzing nascent products on surfaces in a state-selective way. In the future, the energy distributions of nascent products will be analyzed before desorption. This development is not impossible, since the vibration frequency is much shorter than the energy relaxation time. The application of AR product desorption to surface-structural information is still restricted to a limited extent. Such an analysis will deliver more information when it can be performed at a state-selective level. The present situation of surface reaction dynamics is largely due to the absence of internal energies of desorbing products as a function of the desorption angle. The state-selective analysis of desorbing 
products should be combined with the angle-resolved performance; for example, REMPI techniques should be applied to products after AR procedures. This next-generation analysis becomes important in improving the AR-desorption method because each energy state of desorbing products will show different structural information depending on their energy partitioning.

The relationship of desorption dynamics to site structures will be more elaboratively evaluated by adding the desorption-angle dependence of the internal energies. This dependence will show the mechanism of energy partitioning into the rotational, vibrational, and translational modes in a repulsive desorption event. Furthermore, it will deliver the configuration of transition states immediately before desorption. This correlation has been characterized neither experimentally nor theoretically.

Acknowledgements

One of the authors (TM) thanks the fellowship support of the Royal Society and the hospitality of Professor Michael Bowker and his group members at Cardiff University during the preparation of this review. This work was partly supported by a 1996 Center of Excellence (COE) Special Equipment Program of the Ministry of Education, Sports, and Culture of Japan and by Grant-in-Aid No. 21550004 for General Scientific Research from the Japan Society for the Promotion of Science. 
References

[1] K. Tamaru, in "Dynamic Heterogeneous Catalysis" (Academic Press. London, 1978) p 96.

[2] G. Ertl, in "Catalysis Science and Technology" edits. J. R. Anderson, M. Boudart, (Springer-Verlag, Berlin, 1983) p 209.

[3] J. Horiuchi, J. Mol. Catal. A. Chem. 199 (2003) 199-234.

[4] V. P. Zhdanov, Surf. Sci. Rep. 45 (2002) 231-326.

[5] G. Ertl, Catal. Rev. Sci. Eng. 21 (1980) 201-223.

[6] G. Ertl, Adv. in Catal. 45 (2000) 1-69.

[7] P. Casavecchia, N. Balucani, G. G. Volpi, Annu. Rev. Phys. Chem. 50 (1999) 347-376.

[8] J.C. Polanyi, A.H. Zewail, Acc. Chem. Res. 28 (1995) 11-132.

[9] H.S. Taylor, Proc. Roy. Soc. A 108 (1925) 105.

[10] T. Matsushima, Prog. Surf. Sci. 82 (2007) 435-477.

[11] H. Ueba, Prog. Surf. Sci. 55 (1997) 115-179.

[12] A. Gross, Surf. Sci. 500 (2002) 347-367.

[13] W. Brenig, E. Pehlke, Prog. Surf. Sci. 83 (2008) 263-336.

[14] J.C. Polanyi, K.B. Woodall, J. Chem. Phys. 57 (1972) 1574-1586.

[15] K. Liu, Ann. Rev. Phys. Chem. 52 (2001) 139-164.

[16] D. M. Neumark, A. M. Wodtke, G. N. Robinson, C. C. Hayden, Y. T. Lee, J. Chem. Phys. 82 (1985) 3045-3066.

[17] D. M. Neumark, A. M. Wodtke, G. N. Robinson, C. C. Hayden, K. Shobatake, R. K. Sparks, T. P. Schafer, Y. T. Lee, J. Chem. Phys. 82 (1985) 3067-3077.

[18] K. Stark, H.-J. Werner, J. Chem. Phys. 104 (1996) 6515-6530.

[19] J.C. Polanyi, Account. Chem. Research, 5 (1972) 161-168.

[20] T. Takayanagi, S. Sato, Chem. Phys. Lett. 144 (1988)191-193.

[21] C. T. Rettner, Phys. Rev. Lett. 69 (1992) 383-386.

[22] T. A. Germer, J. C. Stephenson, E. J. Heilweil, R. R. Cavanagh, J. Chem. Phys. 98 (1993) 9986-9994.

[23] J. C. Owrutsky, J. P. Culver, M. Li, Y. R. Kim, M. J. Sarisky, M. S. Yeganeh, A. G. Yodh, R. M. Hochstrasser, J. Chem. Phys. 97 (1992) 4421-4427. 
[24] E. Hasselbrink, Curr. Opin. Solid State Mater. Sci. 10 (2007) 192-204.

[25] M. Born, R. Oppenheimer, Ann. Phys. 84 (1927) 457-484.

[26] E. J. Heilweil, R. R. Cavanagh, J. C. Stephenson, J. Chem. Phys. 89 (1988) 5342-5343.

[27] K. Lass, E. Hasselbrink, J. Chem. Phys. 123 (2005) 051102 (4).

[28] H.-C. Chang, G. E. Ewing, Phys. Rev. Lett. 65 (1990) 2125-2128.

[29] R. C. Millikan, J. Chem. Phys. 38 (1963) 2855-2860.

[30] J. Peter Toennies, J. Vac.Sci. \& Technol. A2 (1984) 1055-1065.

[31] H. Nienhaus, H. S. Bergh, B. Gergen, A. Majumdar, W. H. Weinberg, E. W. McFarland, Phys. Rev. Lett. 82 (1999) 446-449.

[32] Wolf-Dieter Schoene, Prog. Surf. Sci. 82 (2007) 161-192.

[33] H. Nienhaus, Surf. Sci. Rep. 45 (2002) 1-78.

[34] H. B. Michaelson, J. Appl. Phys. 48 (1977) 4729-4733.

[35] T. Greber, Surf. Sci. Rep. 28 (1997) 1- 64.

[36] J. J. Thomson, Phil. Mag. 10 (1905) 584.

[37] A. Böttcher, R. Imbeck, A. Morgante, G.Ertl, Phys. Rev. Lett. 65 (1990) 2035-2037.

[38] R. Grobecker, H. Shi, H. Bludau, T. Hertel, T. Greber, A. Böttcher, K, Jacobi, G. Ertl, Phys. Rev. Lett. 72 (1994) 578-581.

[39] L. Hellberg, J. Strömquist, B. Kasemo, B. I. Lundqvist, Phys. Rev. Lett. 74 (1995) $4742-4745$.

[40] B. Kasemo, E. T"ornqvist, J. K. Nørskov, B. I. Lundqvist, Surf. Sci. 89 (1979) 554-565.

[41] A. J. Komrowski, J. Z. Sexton, A. C. Kummel, M. Binetti, O. Weiße, E. Hasselbrink, Phys. Rev. Lett. 87 (2001) 246103 (4).

[42] J. D. White, J. Chen, D. Matsiev, D. J. Auerbach, A. M. Wodtk, Nature, 433 (2005) 503-505.

[43] N. H. Nahler, J. D. White, J. LaRue, D. J. Auerbach, A. M. Wodtke, Science, 321 (2008) 1191-1194.

[44] G. Comsa, R. David, Surf. Sci. Rep. 5 (1985) 145-198.

[45] T. Matsushima, Surf. Sci. Rep. 51 (2003) 1-62.

[46] H.A. Michelsen, C.T. Rettner, D.J. Auerbach, in "Surface Reactions, Springer Series in Surface Sciences,“(Ed.) R.J. Madix (Springer-Verlag, Berlin, 1994) vol. 34, p 185. 
[47] M. Balooch, M.J. Cardillo, D.R. Miller, R.E. Stickney, Surf. Sci. 46 (1974) 358-392.

[48] H.P. Steinrück, A. Winkler, K.D. Rendulic, Surf. Sci.152/153 (1985) 323-327.

[49] T. Matsushima, Chem. Phys. Lett. 155 (1989) 313-316.

[50] A. V. Walker, D. A. King, J. Chem. Phys. 112 (2000) 1937-1945.

[51] Y. Ohno, K. Kimura, M. Bi, T. Matsushima, J. Chem. Phys. 110 (1999) 8221-8224.

[52] Y. Ma, T. Matsushima, K. Shobatake, A. Kokalj, J. Chem. Phys. 124 (2006) 144711 (11).

[53] T. E. Madey, D. E. Ramaker, R. Stockbauer, Ann. Rev. Phys. Chem. 35 (1984) 215-240.

[54] R. H. Stulen, Prog. Surf. Sci. 32 (1989) 1-38.

[55] R.D. Ramsier, J.T. Yates, Jr., Surf. Sci. Rep. 12 (1991) 243-378.

[56] D. Menzel, R. Gomer, J. Chem. Phys. 40 (1964) 1164-1165.

[57] D. Menzel, R. Gomer, J. Chem. Phys. 41 (1964) 3311-3328.

[58] P.A. Redhead, Can. J. Phys. 42 (1964) 886.

[59] J. Wintterlin, R. Schuster, G. Ertl, Phys. Rev. Lett. 77 (1996) 123-126.

[60] B. C. Stipe, M. A. Rezaei, W. Ho, J. Chem. Phys. 107 (1997) 6443-6447.

[61] I. Kobal, A. Kokalj, H. Horino, Y. Ohno, T. Matsushima, Trends in Chem. Phys. 10 (2002) 139-178.

[62] T. Hirayama, I. Arakawa, J. Phys.: Condens. Matter, 18 (2006) 1563-1580.

[63] P.R. Antoniewicz, Phys. Rev. B 21 (1980) 3811-3815.

[64] C.T. Rettner, D.J. Auerbach, Phys. Rev. Lett. 74 (1995) 4551-4554.

[65] P.H. Kobrin, G.A. Schick, J.P. Baxter, N. Winograd, Rev. Sci. Instrum. 57 (1986) 1354-1362.

[66] M. Kobayashi, Y. Tuzi, J. Vac. Sci. Technol. 16 (1979) 685-688.

[67] G. Comsa, R. David, B. J. Schumacher, Rev. Sci. Instrum. 52 (1981) 789-796.

[68] A. Hodgson, Prog. Surf. Sci. 63 (2000) 1-61.

[69] H. Uetsuka, K. Watanabe, K. Kunimori, Surf. Sci. 363 (1996) 73-78.

[70] T. Yamanaka, T. Matsushima, Rev. Sci. Inst. 78 (2007) 034105 (6).

[71] A. V. Zeigarnik, Kinet. \& Catal. 44 (2003) 250-265.

[72] H. Horino, S. Liu, A. Hiratsuka, Y. Ohno, T. Matsushima. Chem. Phys. Lett. 341 (2001) 419-424.

[73] K. Imamura, H. Horino, I. Rzeznicka, I. Kobal, A. Kokalj, Y. Ohno, B. E. Nieuwenhuys, A. 
Hiratsuka, T. Matsushima, Surf. Sci. 566/568 (2004) 1076-1081.

[74] H. H. Huang. C.S. Seet, Z. Zou, G.Q. Xu, Surf. Sci. 356 (1996) 181-188.

[75] V. P. Zhdanov, T. Matsushima, Surf. Sci. 601 (2007) 2373-2377.

[76] V. P. Zhdanov, T. Matsushima, Surf. Sci. 583 (2005) 253-264.

[77] Y.-S. Ma, T. Matsushima, Catal. Today, 111 (2006) 302-310.

[78] H. Goldstein; Classical Mechanics, 2nd Ed. (Addison-Wesley, Reading, Mas. 1980) p.143.

[79] K. Watanabe, A. Kokalj, Y. Inokuchi, I. I. Rzeznicka, K. Ohshimo, N. Nishi, T. Matsushima, Chem. Phys. Lett. 406 (2005) 474-478.

[80] K. Watanabe, A. Kokalj, H. Horino, I. I. Rzeznicka, T. Takahashi, N. Nishi, T. Matsushima, Jpn. J. Appl. Phys. Part 1, 45 (3B) (2006) 2290-2294.

[81] S. Haq, A. Hodgson, Surf. Sci. 463 (2000) 1-10.

[82] A. Kokalj, I. Kobal, T. Matsushima, J. Phys. Chem. B 107 (2003) 2741-2747.

[83] A. Kokalj, T. Matsushima, J. Chem. Phys. 122 (2005) 34708 (10).

[84] T. Matsushima, Phys. Chem. Chem. Phys. 9 (2007) 3031-3042.

[85] K. Christmann, M. Ehsasi, Appl. Phys. A Solid \& Surf. 44 (1987) 87-91.

[86] K. Christmann, Surf. Sci. Rep. 9 (1988) 1-163.

[87] T. Matsushima, O. Nakagoe, K. Shobatake, A. Kokalj, J. Chem. Phys. 125 (2006) 133402 (10).

[88] G. Centi, G. E. Arena, S. Perathoner, J. Catal. 216 (2002) 443-454.

[89] T.W. Root, L.D. Schmidt, G.B. Fisher, Surf. Sci. 134 (1983) 30-45.

[90] E. Ozensoy, C. Hess, D.W. Goodman, J. Am. Chem. Soc. 124 (2002) 8524-8525.

[91] E. Ozensoy, D.W. Goodman, Phys. Chem. Chem. Phys. 6 (2004) 3765-3778.

[92] M. Ikai, H. He, C.E. Borroni-Bird, H. Hirano, Ken-ichi Tanaka, Surf. Sci. Lett. 315 (1994) L973-L976.

[93] M. Ikai and Ken-ichi Tanaka, J. Phys. Chem. B 103 (1999) 8277-8282.

[94] Se H. Oh, G.B. Fisher, J.E. Carpenter, D W. Goodman, J. Catal. 100 (1986) 360-376.

[95] M.J.P. Hopstaken, J.W. Niemantsverdriet, J. Phys. Chem. B, 104 (2000) 3058-3066.

[96] T. Matsushima, J. Phys. Chem. C 111 (2007) 6422-6427.

[97] I. I. Rzeznicka, Y.-S. Ma, G. Cao, T. Matsushima, J. Phys. Chem. B, 108 (2004) 
14232-14243.

[98] T. Matsushima, Hetero. Chem. Rev. 2 (1995) 51-68.

[99] W. van Willigen, Phys. Lett. 28A (1968) 80-81.

[100] T. Yamanaka, T. Matsushima, Phys. Rev. Lett. 100 (2008) 026104(4).

[101] T. Yamanaka, J. Chem. Phys. 128, (2008) 171102 (4)

[102] M. G. Moula, S. Wako, M. U. Kislyuk, Y. Ohno, T. Matsushima, Surf. Sci. \& Catal. Stu. 132 (2001) 701-704.

[103] R. P. H. Gasser; in "An introduction to chemisorption and catalysis by metals",

(Clarendon Press, Oxford, 1985) Chapter 1.

[104] T. Yamanaka, Phys. Chem. Chem. Phys. 10 (2008) 5429-5442.

[105] T. Yamanaka, e-J. Surf. Sci. \& Nanotech. 6 (2008) 180-190.

[106] V.R. Dhank,G. Comelli,G. Paolucci,K.C. Prince,R. Rosei, Surf. Sci. 260 (1992) L24-L27. [107] I.I. Rzeznicka, T. Matsushima, Chem. Phys. Lett. 377 (2003) 279-285

[108] Md. G. Moula, S. Wako, G. Cao, K. Kimura, Y. Ohno, I. Kobal, T. Matsushima, Phys. Chem. Chem. Phys. 1(1999) 3677-3683

[109] S. Wako, M. G. Moula, G. Cao, K. Kimura I. Kobal, Y. Ohno, T. Matsushima, Langmuir, 16(2000) 2689-2696.

[110] A.R. Burns, E.B. Stechel, D.R. Jennison, in Desorption Induced by Electron Transitions DIET V in Springer Series in Surface Sciences, Vol.31 (1993) p173-177, Eds. A.R. Burns, E.B. Stechel, D.R. Jennison.

[111] A.R. Burns, Surf. Sci. 280 (1995) 349-358.

[112] Ken Snowdon, in Desorption Induced by Electron Transitions DIET V in Springer Series in Surface Sciences Vol.31, Eds. A.R. Burns, E.B. Stechel, D.R. Jennison. (Springer, New York 1993) p116-122.

[113] A.R. Burns, E.B. Stechel, D.R. Jennison,, Surf. Sci. 280 (1995) 359-368.

[114] M. Wilde, K. Fukutani, Y. Murata, M. Kampling, K. Al-Shamery, H. J. Freund, Surf. Sci. 427/428 (1999) 27-33.

[115] A. Alavi, P. Hu, T. Deutsch, P. L. Silvestrelli, J. Hutter, Phys. Rev. Lett. 80 (1998) 3650-3653.

[116] S. Han, T. Matsushima, Phys. Chem. Chem. Phys .7 (2005) 651-658 
[117] S. Han, Y.-S. Ma, T. Matsushima, J. Chem. Phys. 123 (2005) 094702 (9)._

[118] G. Cao, M. G. Moula, Y. Ohno, T. Matsushima, J. Phys. Chem. B103 (1999) 3235-3241.

[119] I.I. Rzeznicka, M.G. Moula, L.M. Garza, Y. Ohno, T. Matsushima, J. Chem. Phys. 119 (2003) 9829-9841. 
Table 1 Relaxation time ( $\tau$ ) of vibrationally excited $\mathrm{CO}$ on various surfaces

\begin{tabular}{lcccccc} 
Surface & $\mathrm{Pt}(111)$ & $\mathrm{Cu}(111)$ & $\mathrm{Rh}_{2}(\mathrm{CO})_{4} / \mathrm{SiO}_{2}$ & $\mathrm{Si}(100)$ & $\mathrm{NaCl}$ & gaseous \\
Temp./K & 150 & 93 & (room temperature) & $\sim 100$ & 22 & 286 \\
\hline$\tau / \mathrm{nsec}$ & 0.0022 & 0.002 & 0.125 & 2.3 & 4300 & 33000 \\
$-{ }^{2}$ & & & & & & \\
(ref.) & {$[22]$} & {$[23]$} & {$[26]$} & {$[27]$} & {$[28]$} & {$[29]$}
\end{tabular}


Figure Captions

Fig. 1 Variations of potential energy of the $\mathrm{H}_{2}+\mathrm{F}$ reaction system along the reaction coordinate for a linear H-H-F form. The reaction path coordinate on the abscissa is defined as the difference of the sum of H-F and $\mathrm{H}-\mathrm{H}$ bond distances from their smallest value. One Bohr is $0.53 \AA$. The zero point energy of the reaction system, as well as the vibrational energy levels $\left(v^{\prime}=0,1,2\right.$, and 3) of product HF, is shown in $\mathrm{kcal} / \mathrm{mol}$ units. (Ref. 18)

Fig. 2 (a) HF product angular distribution for the $\mathrm{F}+\mathrm{p}-\mathrm{H}_{2}$ reaction at a collision energy of 1.84 $\mathrm{kcal} / \mathrm{mol}$ as a function of the laboratory scattering angle $\Theta$, and Newton diagram. Both the data and calculated best-fit laboratory distributions for each vibrational state and the total sum of all contributions are shown. (b) Center-of-mass velocity flux contour map with a three-dimensional perspective. The angle indicated as $0^{\circ}$ refers to the direction of the incident $\mathrm{F}$ atom c.m. velocity. Lines are spaced linearly along the three-dimensional contour according to the scattering intensity. (Ref. 16)

Fig. 3 (a) Potential energy surface for the collinear $\mathrm{F}+\mathrm{H}_{2} \rightarrow \mathrm{FH}+\mathrm{H}$ reaction. (b) Potential energy as a function of the $\mathrm{H}_{\mathrm{a}}-\mathrm{H}_{\mathrm{b}}-\mathrm{F}$ bond angle at the saddle point, where $\mathrm{H}_{\mathrm{a}}$ and $\mathrm{H}_{\mathrm{b}}$ stand for the terminal and central hydrogen atom. The energy levels shown are the vibrational quantum states numerically calculated. (Refs. 18 and 20)

Fig. 4 Chemicurrent response recorded from a $\mathrm{Ag} / \mathrm{n}-\mathrm{Si}(111)$ diode with a $75 \AA$ thick $\mathrm{Ag}$ film when the Ag surface is exposed to a hydrogen atom beam. The inset shows the principle of detection of hot electrons with a thin-semiconductor (Schottky) diode, where the Fermi level, $E_{\mathrm{F}}$, the conduction band minimum (CBM) and the valence band maximum (VBM) are shown. The barrier height $(\Phi)$ reaches the work function difference between $\mathrm{Ag}$ and $\mathrm{Si}$, showing bent conduction and valence bands. The chemical adsorption creates an excited electron-hole pair. The hot electron may travel in a ballistic way through the thin film, traverse the Schottoky barrier, and be detected as a current. (Ref. 31) 
Fig. 5 Variations of the current due to exoelectrons and the work function $(\Psi)$ curve upon stepwise exposure of a Cs film with about $3 \mathrm{ML}$ thickness at $220 \mathrm{~K}$ to an $\mathrm{O}_{2}$ pressure of $4 \times 10^{-7}$ Pa. The oxide states were examined by XPS. (Ref. 37) .

Fig. 6 Principle behind the apparatus of angle-resolved product desorption measurements incorporating time-of-flight techniques. The reaction chamber is an ordinary UHV apparatus with standard facilities for surface analysis and a mass spectrometer. Either the chopper house or the reaction chamber must be evacuated with a large pumping rate. A pseudo-random chopper blade for cross-correlation time-of-flight techniques is drawn. Red ellipses show typical places used for production detection in angle-resolved and non-angle-resolved ways.

Fig. 7 (a) Crystal azimuth $\phi$ and desorption angle $\theta$ used in experiments on $\operatorname{Pd}(110)$. The signal intensity presentation is also drawn. (b) New angle system used to present the three-dimensional distribution of desorbing $\mathrm{N}_{2}$ in the form of a power series of two polar angles $(\alpha, \beta)$ defined in orthogonal planes. The plane at a fixed $\alpha$ value is shown as crosshatched.

Fig. $8(a, b)$ AR-TPD spectra of ${ }^{15} \mathrm{~N}_{2}$ from $\mathrm{Pd}(110)$ exposed to ${ }^{15} \mathrm{~N}_{2} \mathrm{O}$ at $90 \mathrm{~K}$. The desorption angle is in the plane along the [001] direction. (a) $0.03 \mathrm{ML}^{15} \mathrm{~N}_{2} \mathrm{O}$, and (b) $0.08 \mathrm{ML}$. The heating rate was $0.6 \mathrm{~K} / \mathrm{s}$. The signal was corrected by subtraction of the ${ }^{15} \mathrm{~N}_{2} \mathrm{O}$ fragmentation. The curves were deconvoluted into four Gaussian peaks. Angular distributions of desorbing ${ }^{15} \mathrm{~N}_{2}$ in the plane along the [001] direction, (c) $\beta_{4}-\mathrm{N}_{2}$, (d) $\beta_{3}-\mathrm{N}_{2}$ and (e) $\beta_{3}$ and $\beta_{1}-\mathrm{N}_{2}$. The fitting curves are drawn by the inserted equations. The solid line indicates the sum of all the components. The intensity presentation on polar coordinates is drawn on the upper panel. (Ref. 72)

Fig. 9 Spatial distribution of desorbing product $\mathrm{N}_{2}$; (a) Angular distributions of desorbing ${ }^{15} \mathrm{~N}_{2}$ at different crystal azimuths $\left(\phi=0^{\circ}, 18^{\circ}\right.$ and $\left.40^{\circ}\right)$ in the steady-state ${ }^{15} \mathrm{~N}_{2} \mathrm{O}$ reduction on $\operatorname{Pd}(110)$ at $P_{\mathrm{N} 2 \mathrm{O}}=4.4 \times 10^{-4} \mathrm{~Pa}, P_{\mathrm{CO}}=0.7 \times 10^{-4} \mathrm{~Pa}$, and $T_{\mathrm{S}}=520 \mathrm{~K}$. The signal was plotted in a common scale. The crystal azimuth $\phi$ is defined in the inset. The solid curves are simulated by 
the inserted equations. (b) The resultant ${ }^{15} \mathrm{~N}_{2}$ distribution on $\operatorname{Pd}(110)$ is shown on 3-D coordinates as well as the best-fitted equation. This figure was drawn with consideration to the symmetric distribution around the collimation axis. (Ref. 52)

Fig. $10{ }^{15} \mathrm{NO}$ reduction on $\mathrm{Rh}(100)$; Surface temperature dependence of $\mathrm{AR}-{ }^{15} \mathrm{~N}_{2}$ signals at the surface normal and $70^{\circ}$ off normal toward the [001] direction in a steady-state ${ }^{15} \mathrm{NO}+\mathrm{D}_{2}$ reaction. The signals observed in the direction of the increasing surface temperature are designated by closed symbols, and those in the downward direction, by open symbols. The data in the range of $400-800 \mathrm{~K}$ are expanded in the inset. The angular distributions of desorbing ${ }^{15} \mathrm{~N}_{2}$ at $T_{\mathrm{S}}=664 \mathrm{~K}$ and $820 \mathrm{~K}$ are shown on the right column. A typical deconvolution is shown by broken curves. (Ref. 84)

Fig. 11 Infrared emission spectra from the product $\mathrm{CO}_{2}$ in $\mathrm{CO}$ oxidation on $\mathrm{Pd}(111)$ observed at the surface normal (a) and $30^{\circ}$ off normal (b) directions. The total pressure in front of the surface is $0.49 \mathrm{~Pa}$. The $\mathrm{CO} / \mathrm{O}_{2}$ flux ratio is $2: 1$, and the surface temperature is $700 \mathrm{~K}$. Fine lines show the observed spectra with a wave number resolution of $4 \mathrm{~cm}^{-1}$. Under this resolution, the observed emission band becomes broader at higher rotational temperatures. Thick solid lines show the optimum simulated results. The vibrational $\left(T_{\text {vib }}\right)$ and rotational $\left(T_{\text {rot }}\right)$ temperatures used for simulations are listed where $T_{\text {vib }}$ is the average value over all the vibration modes. For comparison, the best fits at $0^{\circ}$ are drawn by the broken lines in the spectrum at $30^{\circ}$. (c) Angle dependence of the rotational and vibrational temperatures. The anti-symmetric vibration temperature $\left(T_{\mathrm{a}}\right)$ was separately estimated from the emission intensity analysis. The other vibration temperatures (symmetric stretching and bending modes) are shown by the average value $\left(T_{\mathrm{sb}}\right)$ because of the absence of separated determinations. (Ref. 101 )

Fig. 12 Surface-phase diagram deduced from the angular distributions of desorbing $\mathrm{CO}_{2}$; Logarithms of $\mathrm{CO}$ pressure $\left(P_{\mathrm{CO}}\right)$ at the kinetic transition (open triangles) and site switching (closed triangles) at a fixed oxygen pressure of $1.3 \times 10^{-2} \mathrm{~Pa}$ are plotted against the reciprocal of the surface temperature $\left(T_{\mathrm{S}}\right)$. Closed circles represent the site switching (distribution changing) observed at $P_{\mathrm{O} 2}=1.3 \times 10^{-5} \mathrm{~Pa}$. The $\mathrm{CO}$ adsorption isotherm at a 0.5 monolayer was determined 
without oxygen. The three-dimensional graphs show the spatial distribution in each region. Split desorption takes place on oxygen-covered $(1 \times 2)$ active areas, whereas the normally directed desorption is on CO-covered $(1 \times 1)$ inhibited areas. The mixed region stands for the presence of the remaining $(1 \times 2)$ areas even above the kinetic transition. (Ref. 119) 


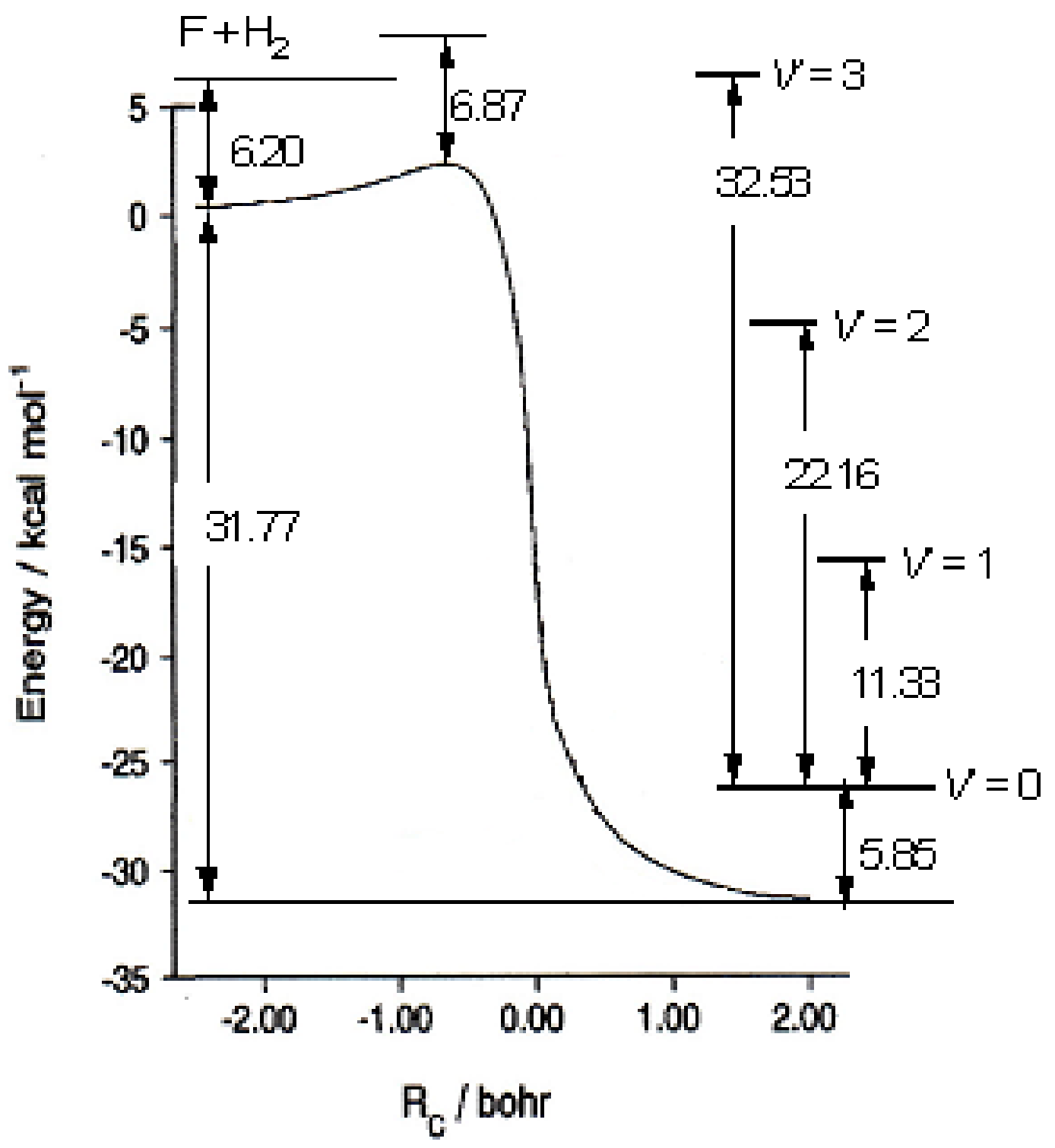

Fig.1 Matsushima 
(b)

(a)

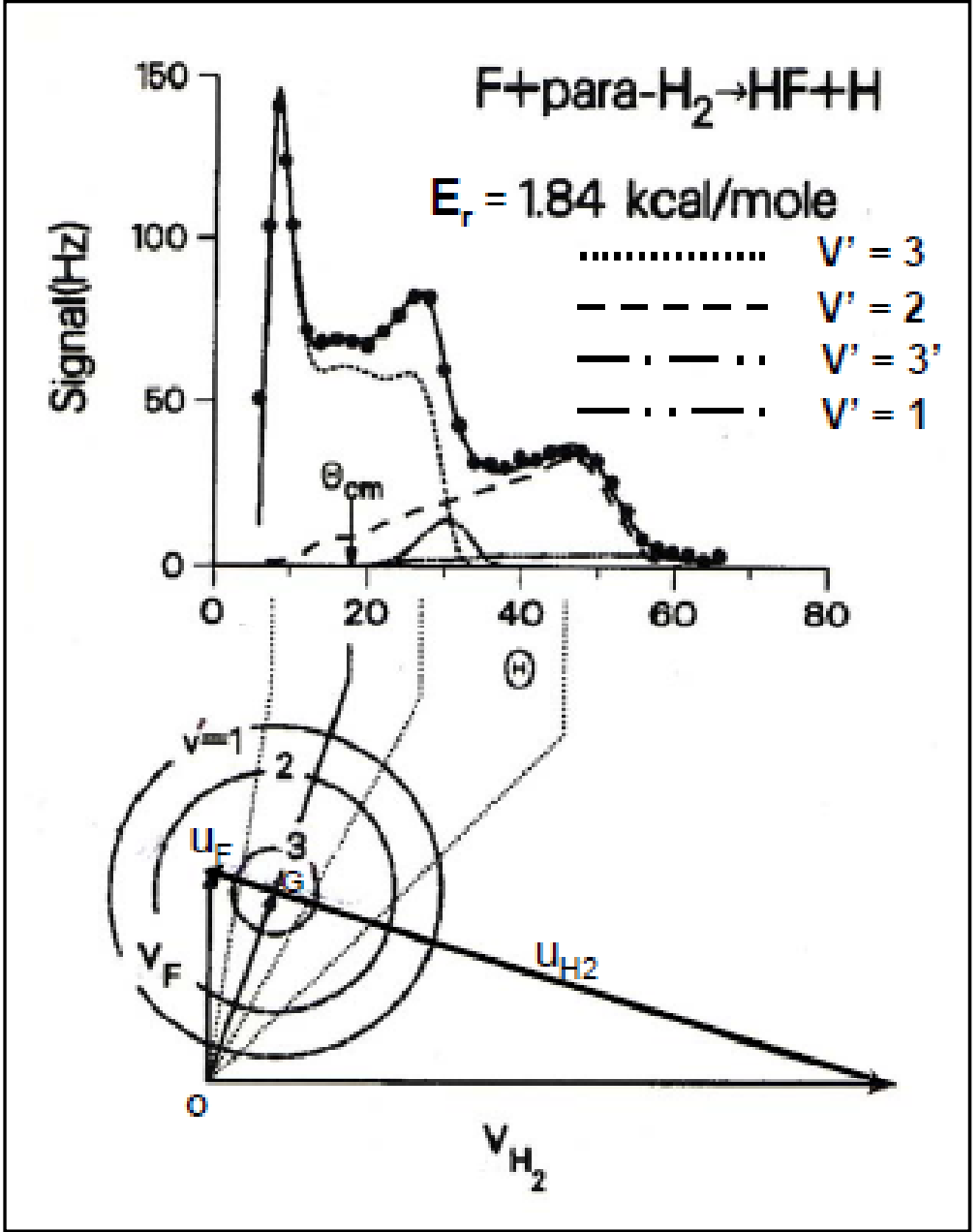

$\mathrm{F}+\mathrm{p}-\mathrm{H}_{2} \rightarrow \mathrm{HF}+\mathrm{H}, 1.84 \mathrm{kcal} / \mathrm{mole}$
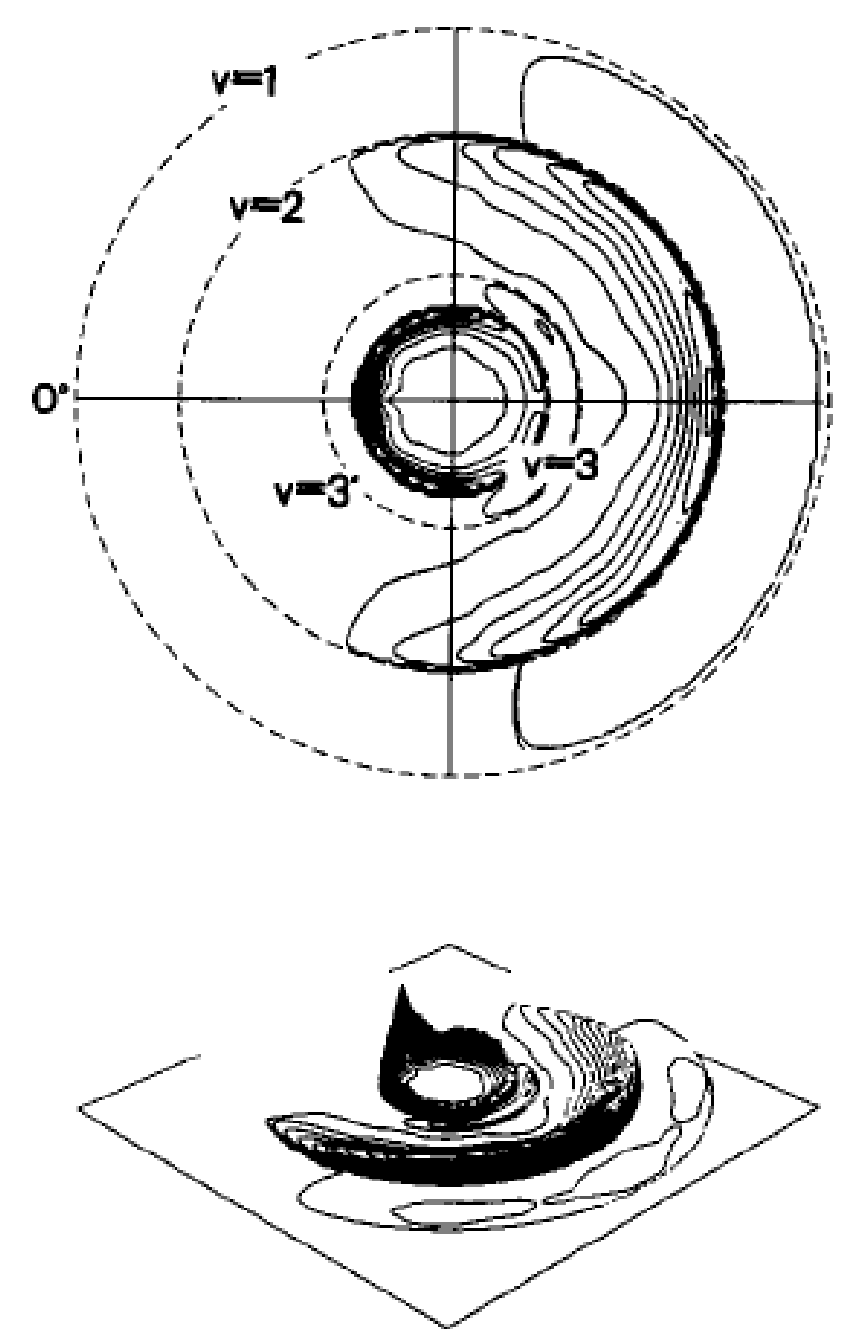

Fig. 2 Matsushima 


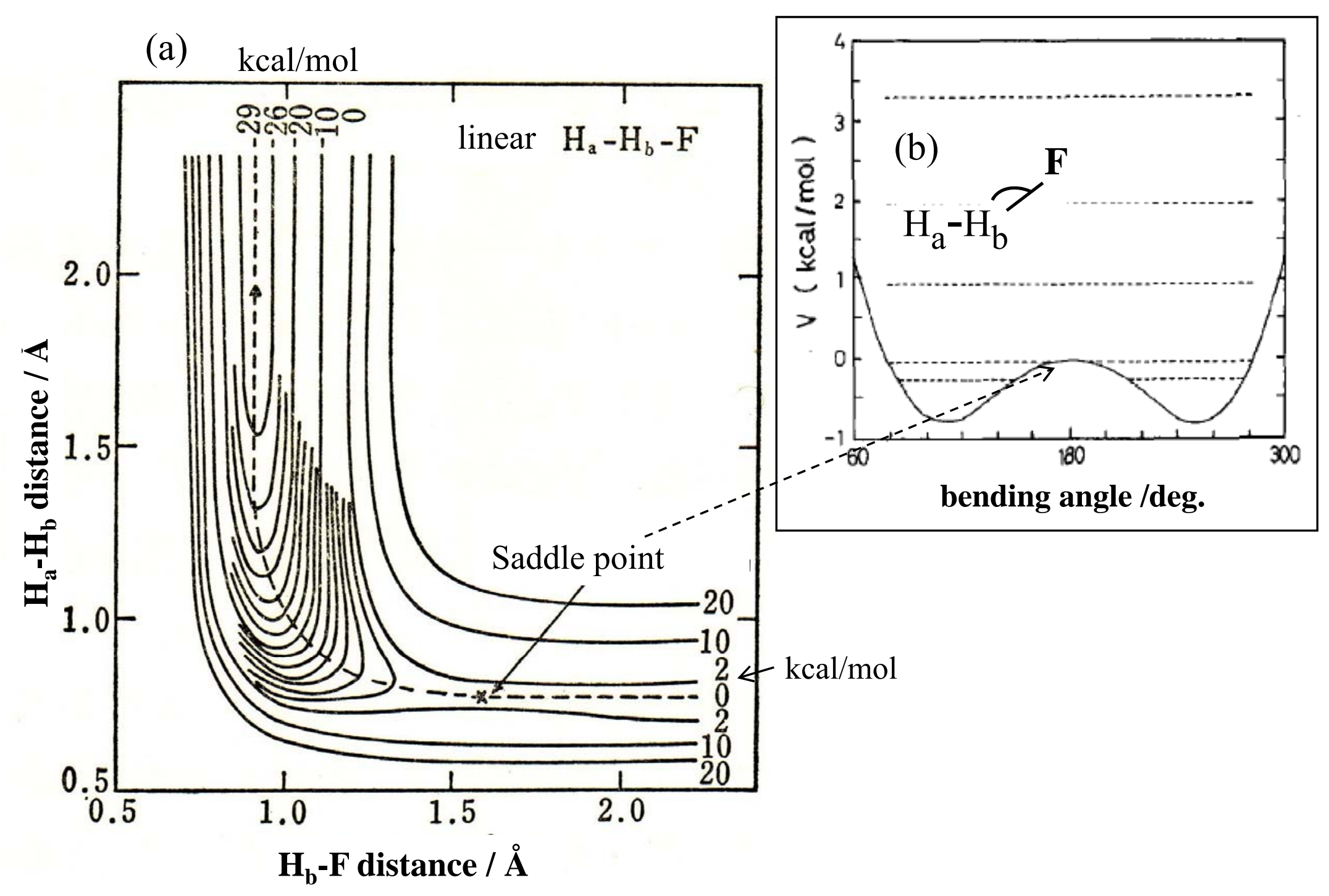

Fig.3 Matsushima 


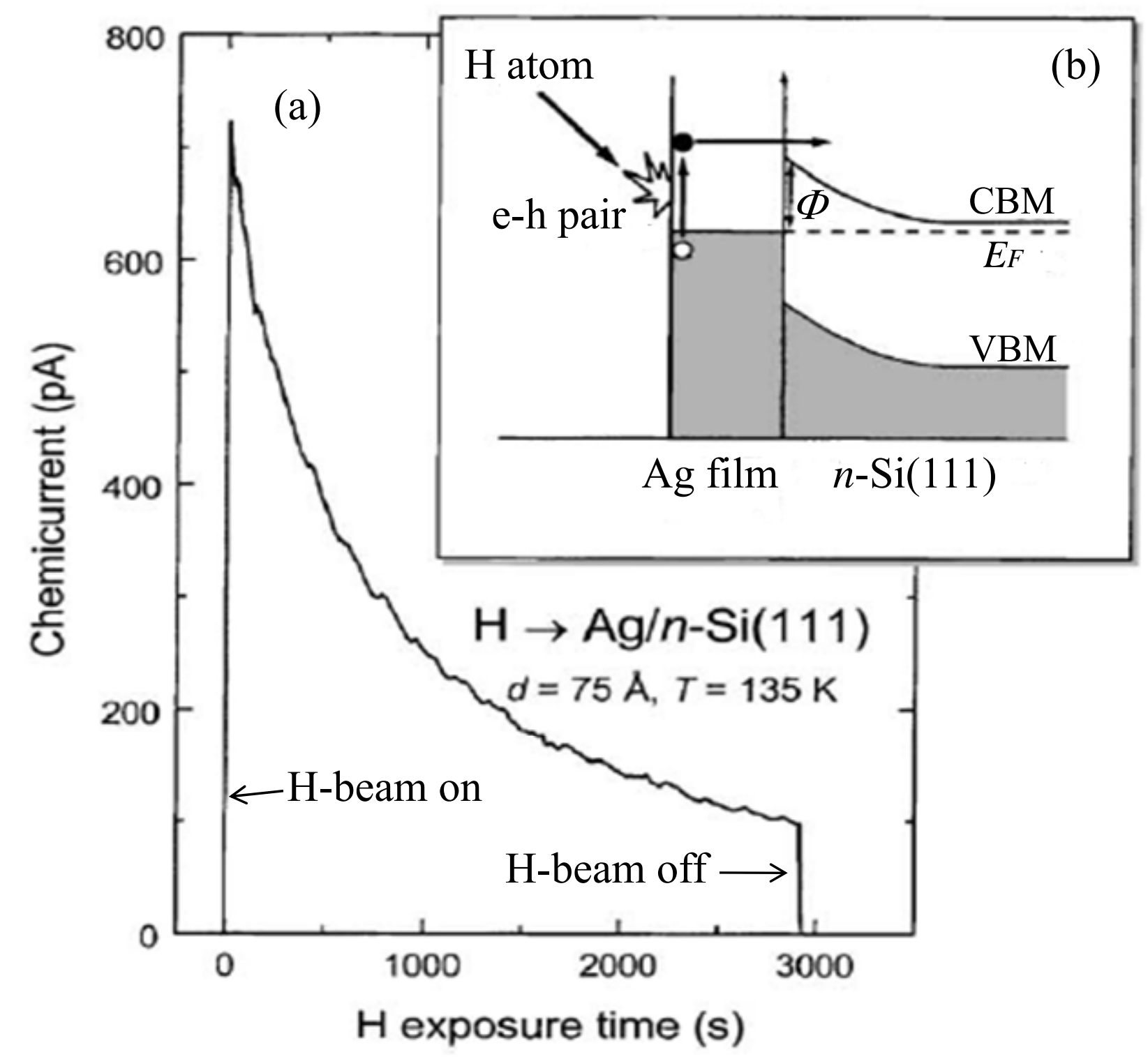

Fig. 4 Matsushima 


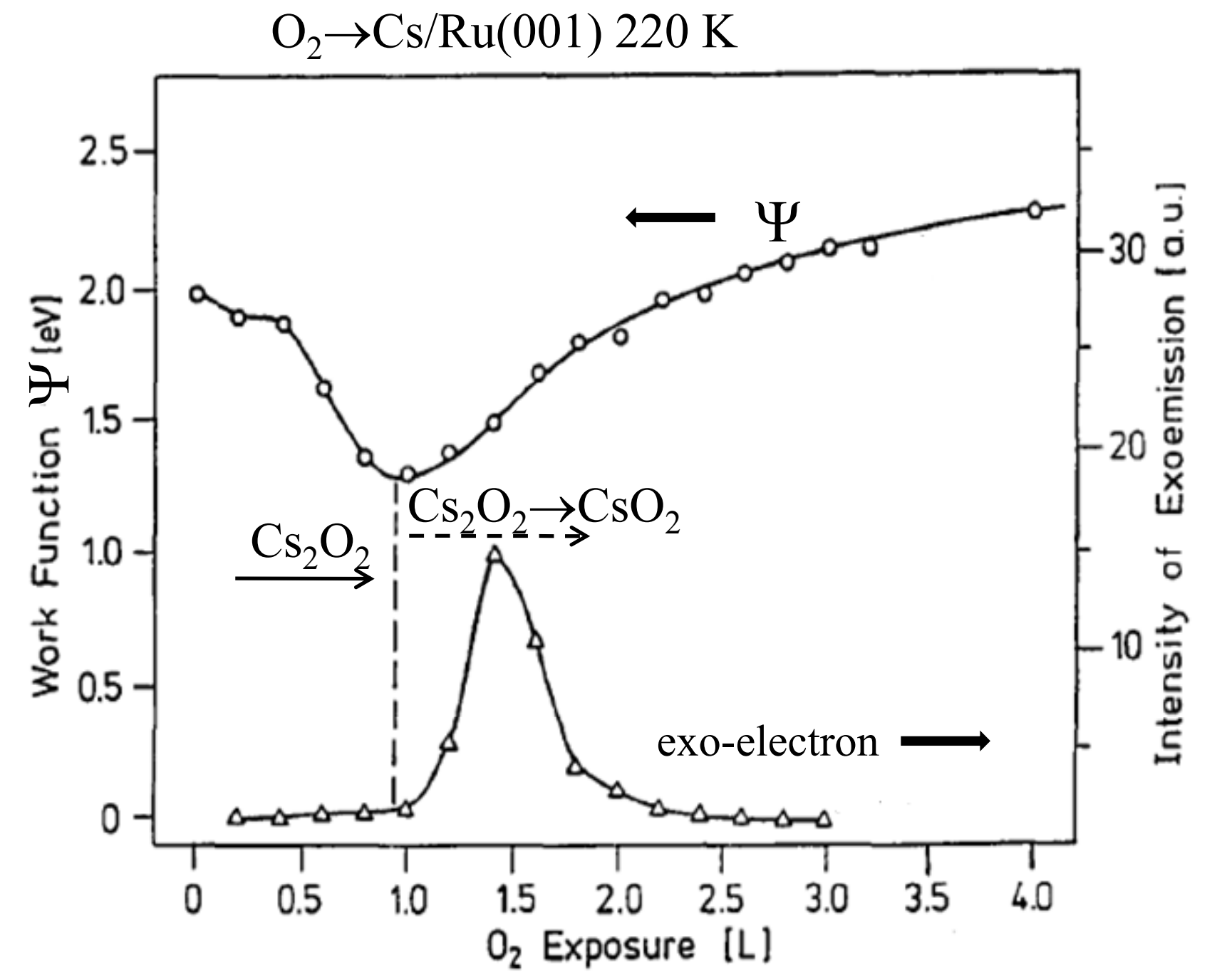

Fig. 5 Matsushima 


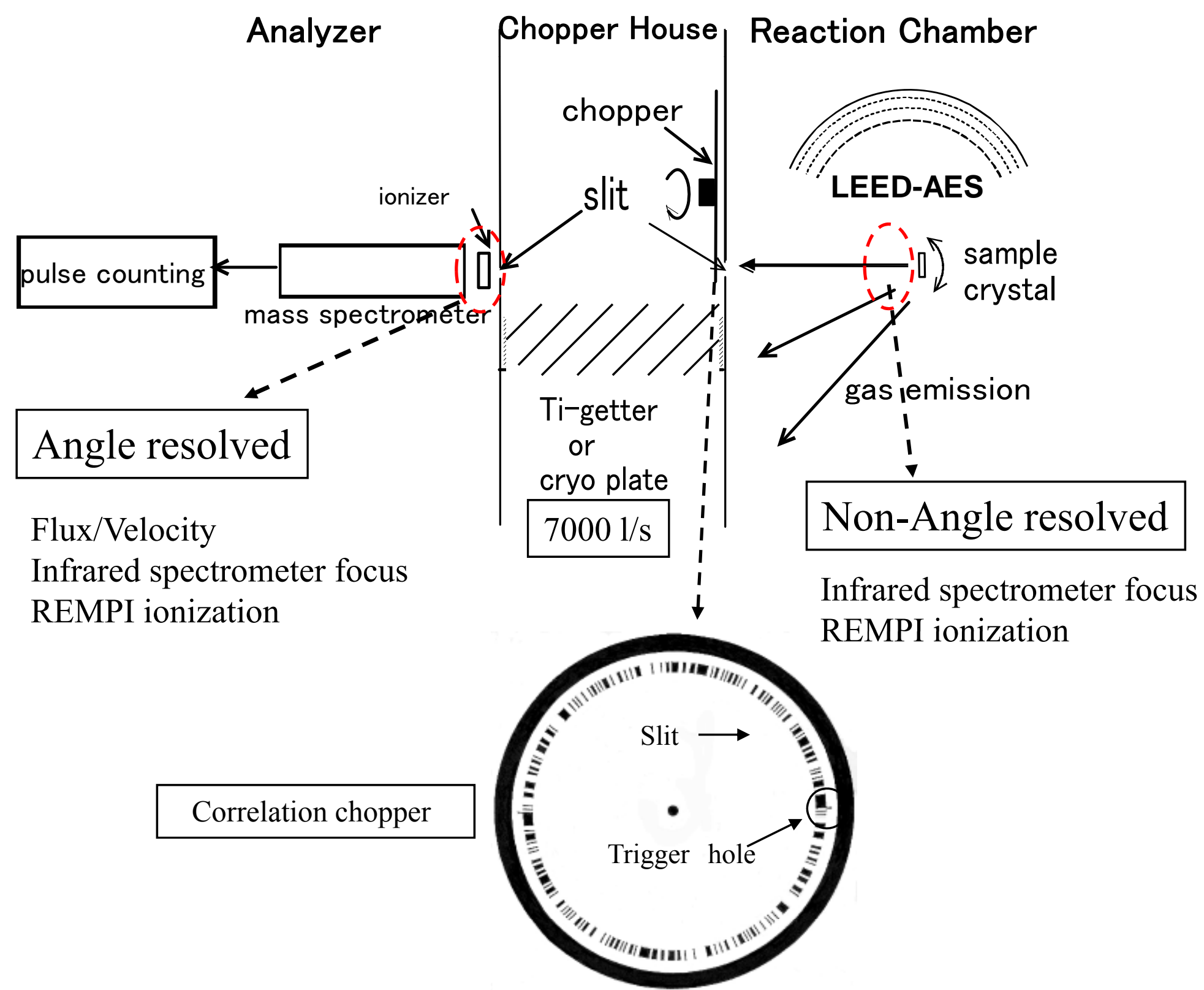

Fig. 6 Matsushima 
(a) Experimental angles

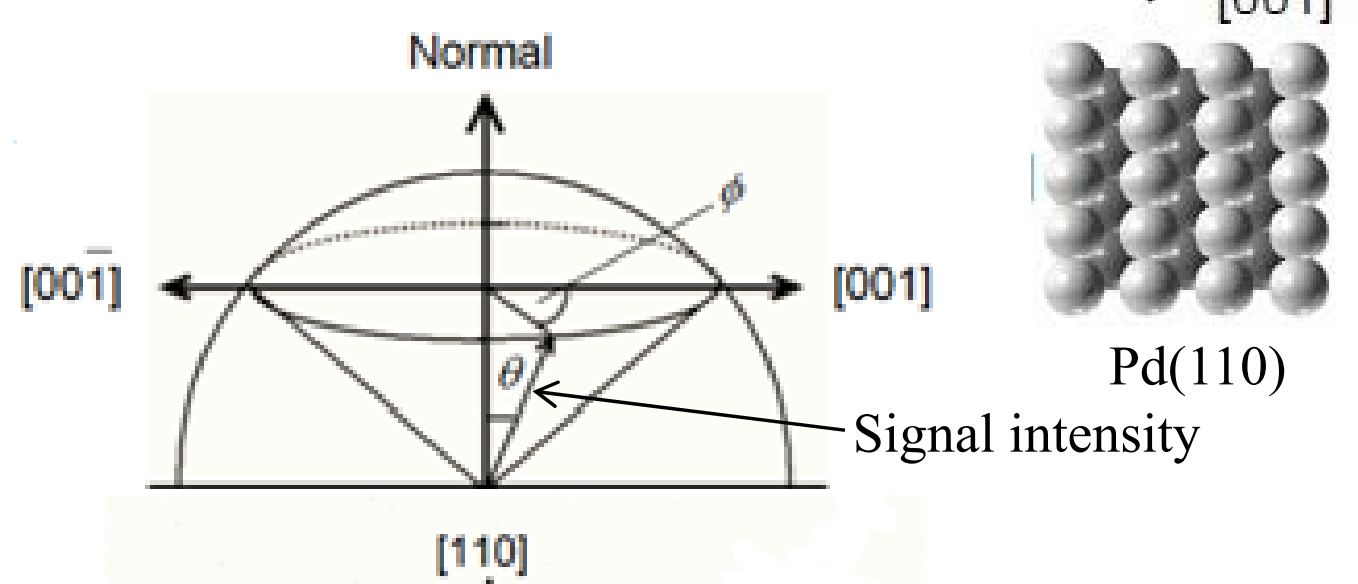

(b) 3D presentation

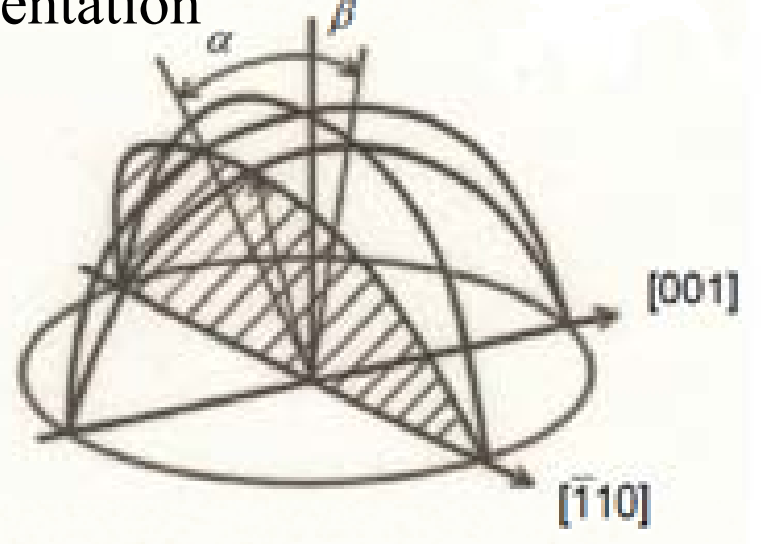



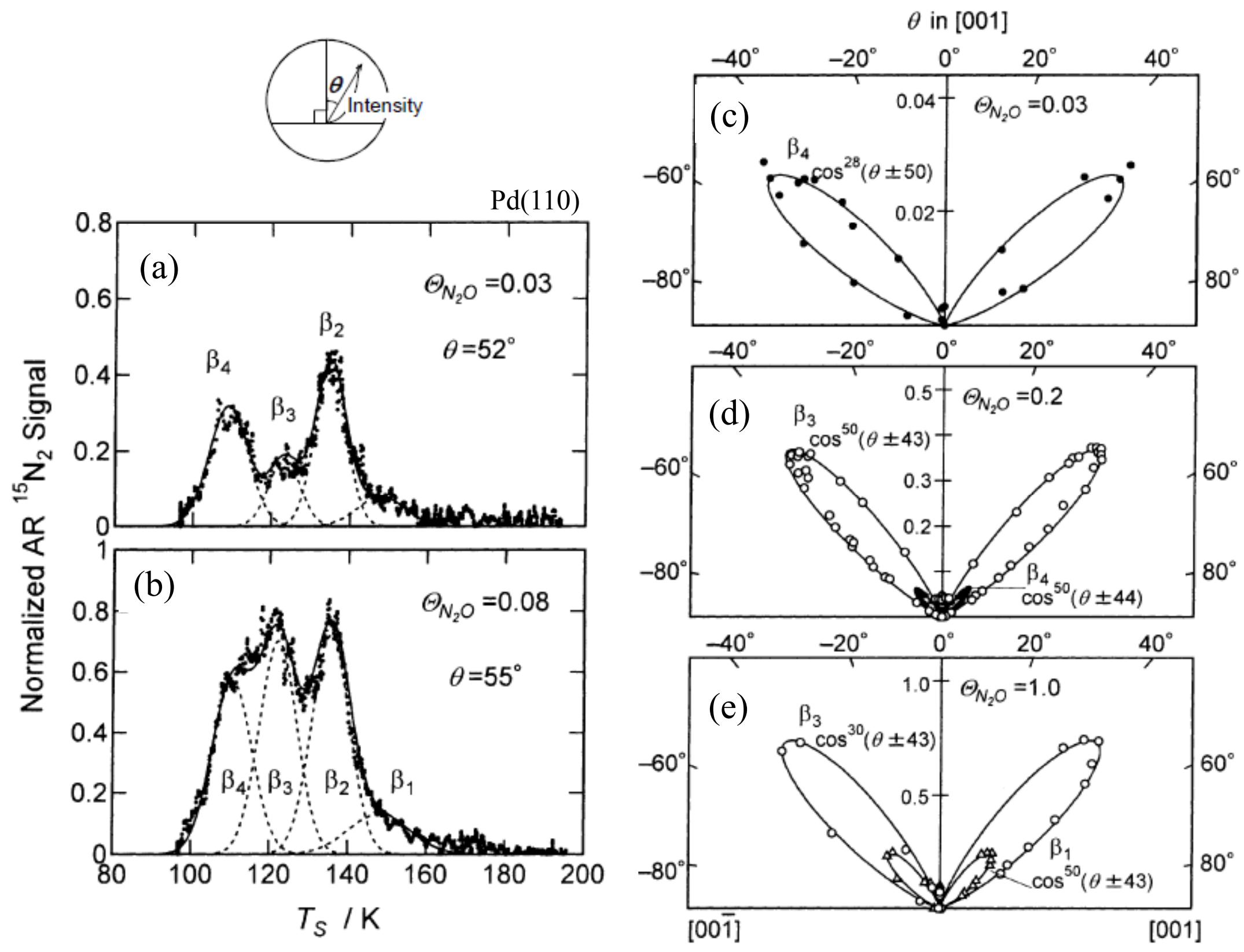

Fig. 8 Matsushima 


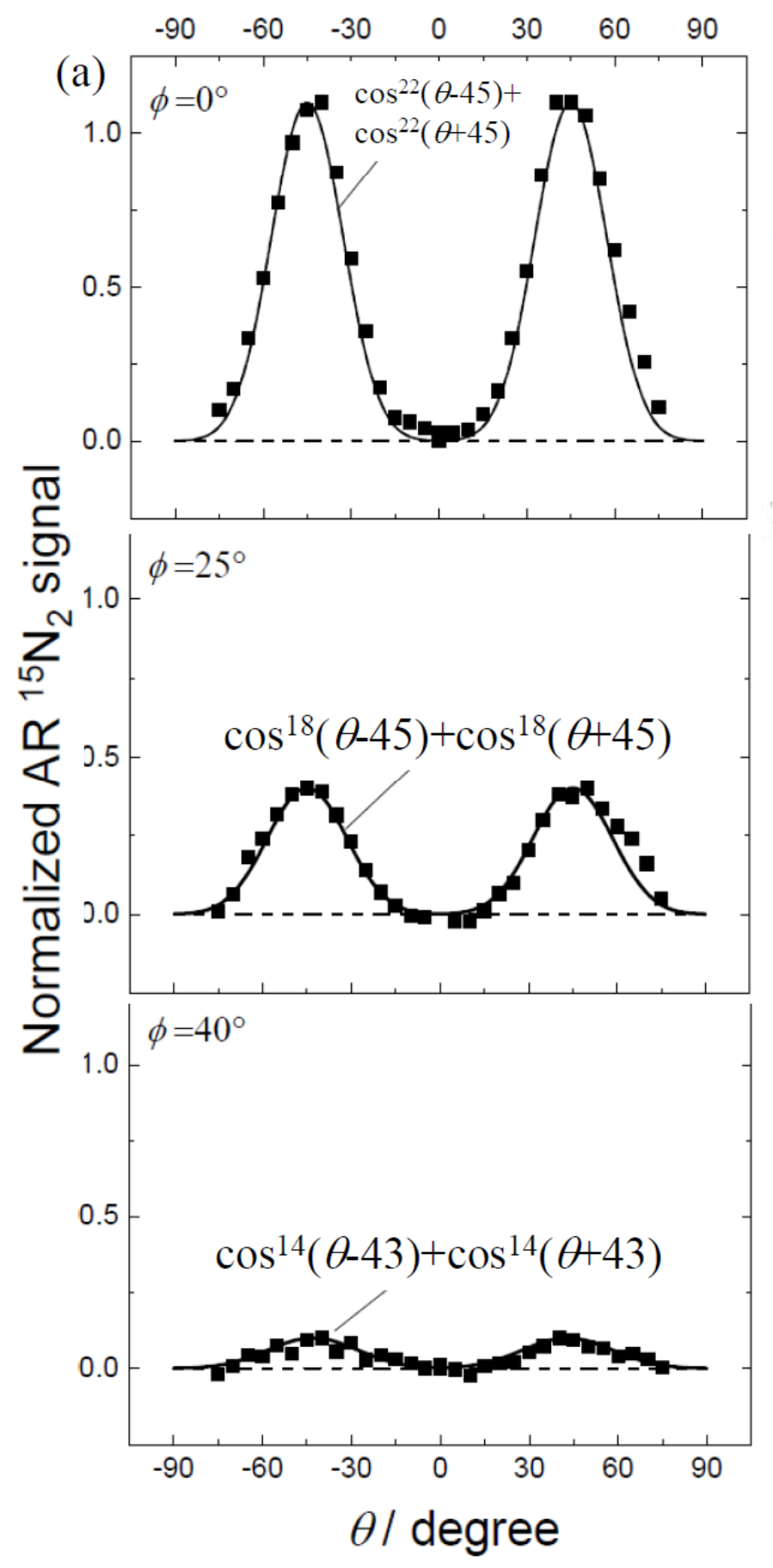

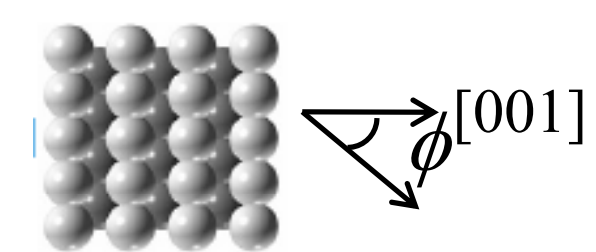

(b)

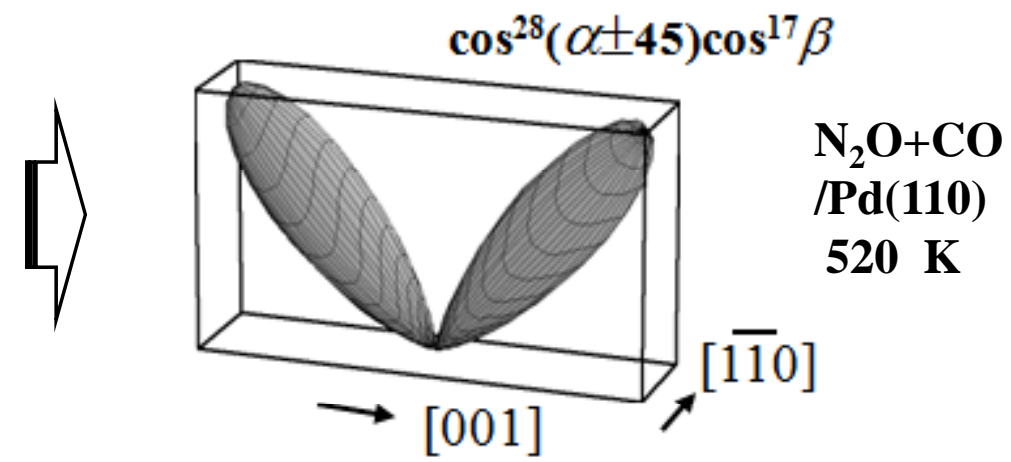

Fig. 9 Matsushima 

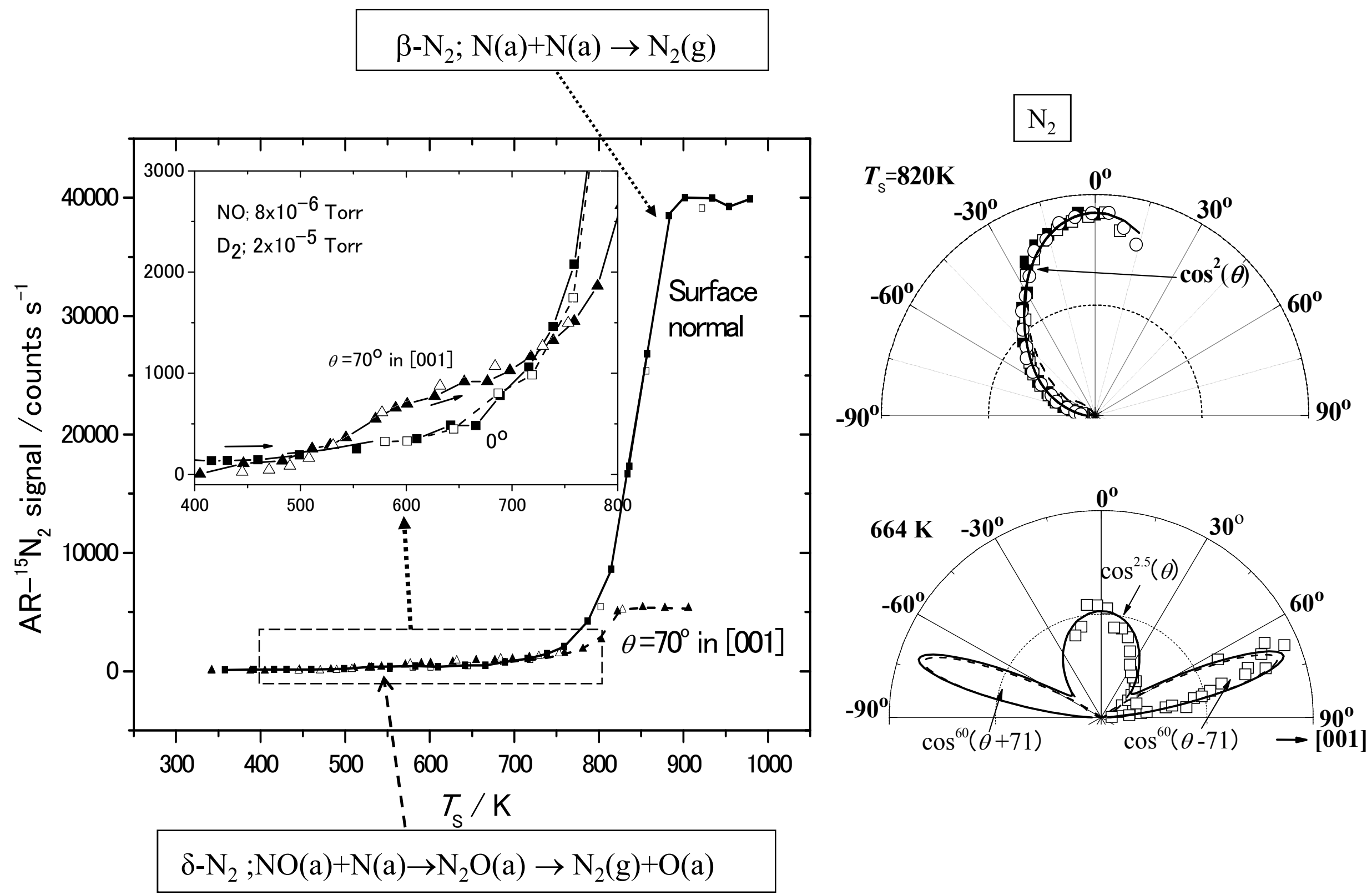

Fig. 10 Matsushima 

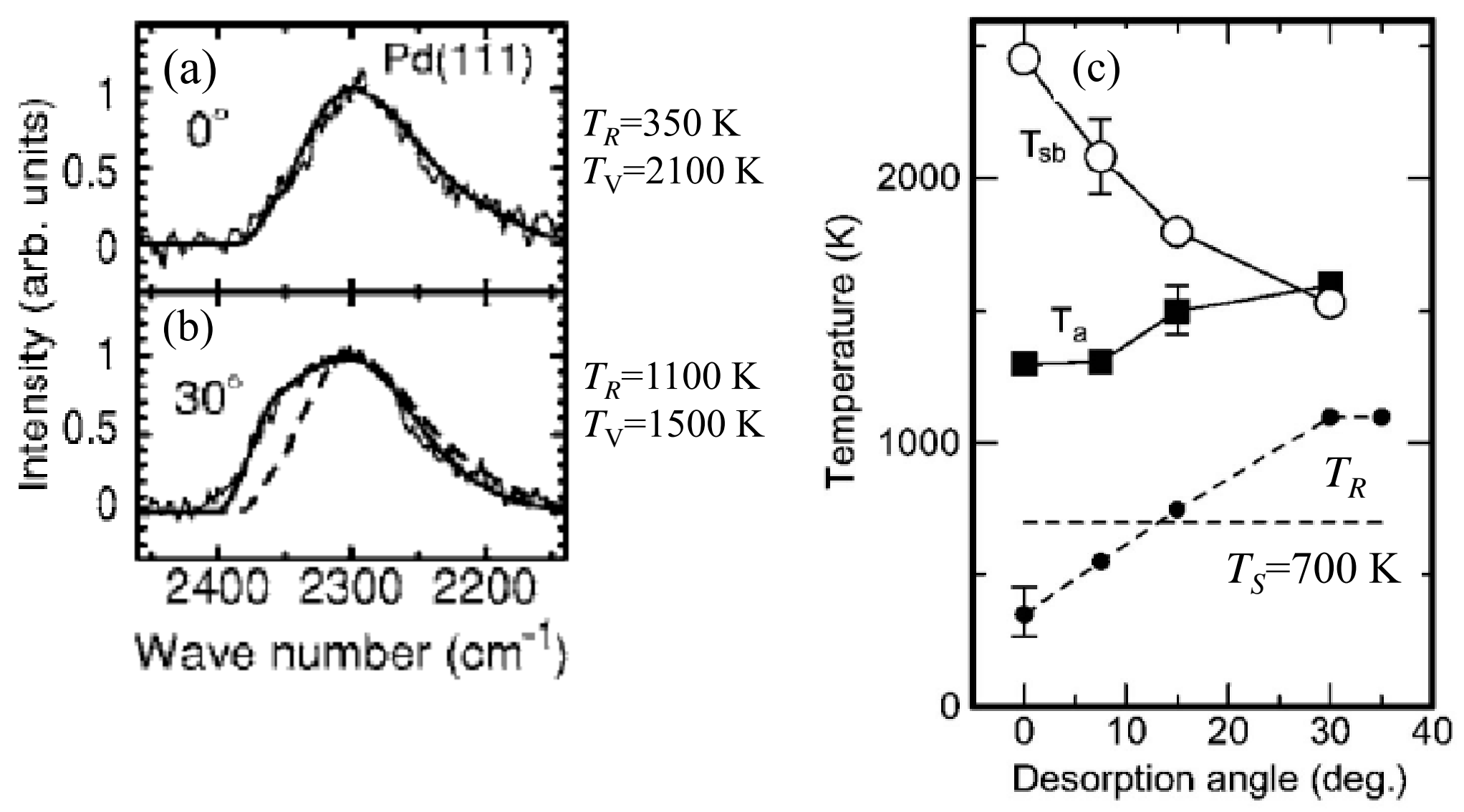

Fig. 11 Matsushima 


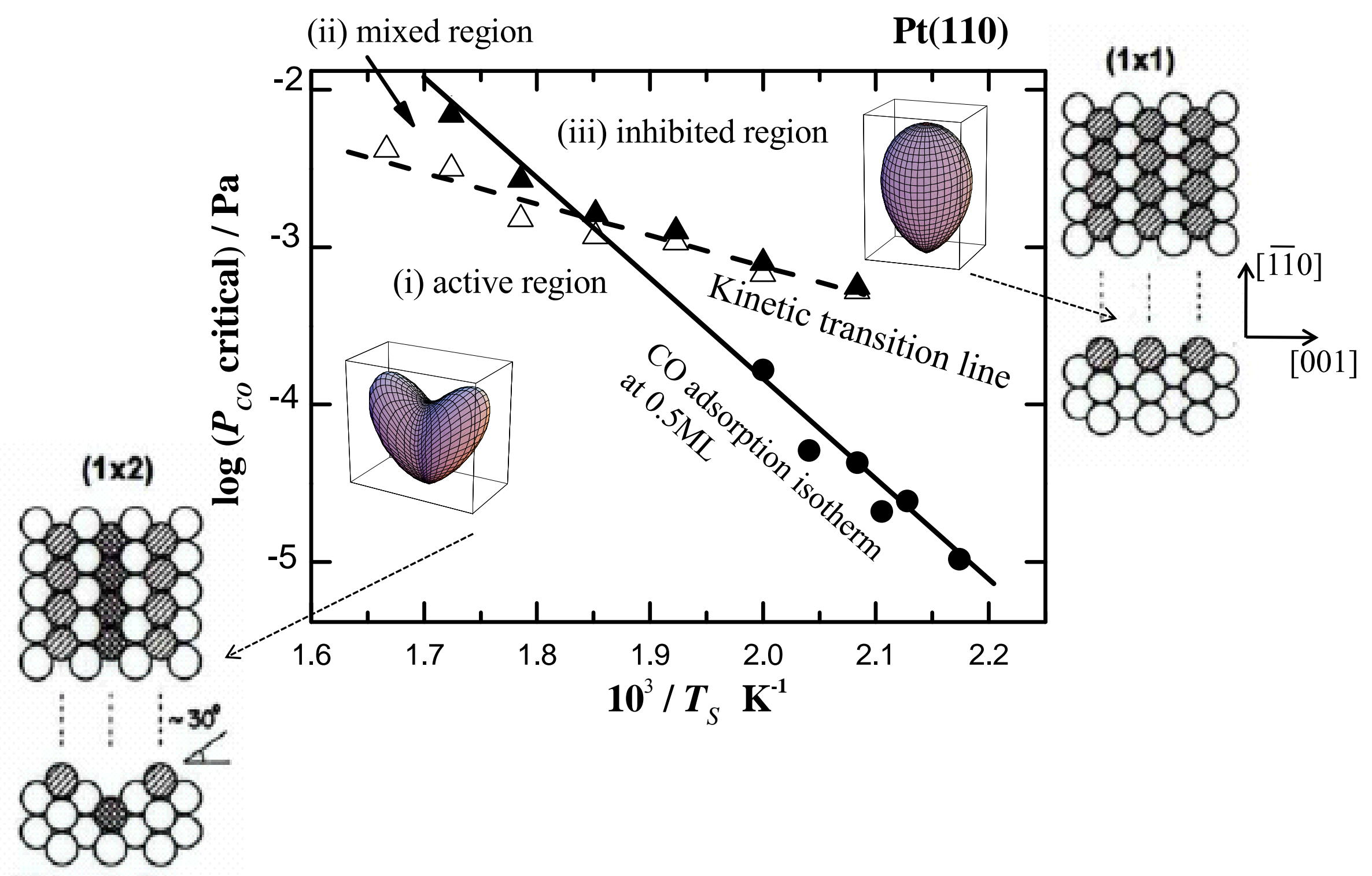

Missing Row

Fig.12 Matsushima 